\title{
Degradation of Chondroitin Sulfate Proteoglycans Potentiates Transplant- Mediated Axonal Remodeling and Functional Recovery after Spinal Cord Injury in Adult Rats
}

\author{
BYUNG G. KIM, HAI-NING DAI, JAMES V. LYNSKEY, MARIETTA MCATEE, \\ AND BARBARA S. BREGMAN* \\ Department of Neuroscience, Georgetown University Medical Center, Washington, DC 20007
}

\begin{abstract}
Transplantation of growth-permissive cells or tissues was used to bridge a lesion cavity and induce axonal growth in experimental spinal cord injury (SCI). Axonal interactions between host and transplant may be affected by upregulation of inhibitory chondroitin sulfate proteoglycans (CSPGs) following various transplantation strategies. The extent of axonal growth and functional recovery after transplantation of embryonic spinal cord tissue decreases in adult compared to neonatal host. We hypothesized that CSPGs contribute to the decrease in the extent to which transplant supports axonal remodeling and functional recovery. Expression of CSPGs increased after overhemisection SCI in adult rats but not in neonates. Embryonic spinal cord transplant was surrounded by CSPGs deposited in host cord, and the interface between host and transplant seemed to contain a large amount of CSPGs. Intrathecally delivered chondroitinase ABC (C'ase) improved recovery of distal forelimb usage and skilled motor behavior after C4 overhemisection injury and transplantation in adults. This behavioral recovery was accompanied by an increased amount of raphespinal axons growing into the transplant, and raphespinal innervation to the cervical motor region was promoted by C'ase plus transplant. Moreover, C'ase increased the number of transplanted neurons that grew axons to the host cervical enlargement, suggesting that degradation of CSPGs supports remodeling not only of host axons but also axons from transplanted neurons. Our results suggest that CSPGs constitute an inhibitory barrier to prevent axonal interactions between host and transplant in adults, and degradation of the inhibitory barrier can potentiate transplant-mediated axonal remodeling and functional recovery after SCI. J. Comp. Neurol. 497:182-198, 2006. (๑) 2006 Wiley-Liss, Inc.
\end{abstract}

Indexing terms: chondroitin sulfate proteoglycans; transplantation; spinal cord injury; axonal remodeling; functional recovery; regeneration

Traumatic injury to the spinal cord leads to a loss of tissue at the injury site resulting in a fluid-filled cavity. Transplantation of growth-permissive scaffolds to bridge the lesion cavity has been one of the essential components in the experimental strategies to repair the injured spinal cord (Horner and Gage, 2000; Schwab, 2002). Transplanted cells or tissues can modify the terrain at the injury site otherwise inhospitable to axonal regeneration into the one conducive to axon growth (Bregman, 1998). Several types of transplants have been tested, including peripheral nerve, Schwann cells, olfactory ensheathing glia, genetically modified fibroblasts, and embryonic spinal cord (Cheng et al., 1996; Xu et al., 1997; Liu et al., 1999; Coumans et al., 2001; Raisman, 2001). The amount of axonal growth into and/or out of transplanted scaffolds,
Grant sponsor: National Institutes of Health (NIH); Grant number: NS 27054; Grant sponsor: International Spinal Research Trust.

Byung G. Kim's current address is Brain Disease Research Center, Ajou University School of Medicine, Suwon, Republic of Korea, 443-721.

James V. Lynskey's current address is Department of Bioengineering, Arizona State University, Tempe, AZ 85287.

*Correspondence to: Barbara S. Bregman, Department of Neuroscience, NRB Rm EP-04, Georgetown University Medical Center, 3970 Reservoir Rd., NW, Washington, DC 20007.

E-mail: bregmanb@georgetown.edu

Received 28 July 2005; Revised 24 December 2005; Accepted 27 January 2006 DOI 10.1002/cne.20980

Published online in Wiley InterScience (www.interscience.wiley.com). 
however, is usually restricted to some extent, and many studies have focused on developing an effective strategy that can achieve more robust axonal growth, such as administration of neurotrophins (Houweling et al., 1998; Bunge, 2001; Coumans et al., 2001).

One of the potential impediments that restrict axonal interaction between transplant and host is the glial scar. Glial scars formed between the host and transplanted tissues or cells may prevent axonal growth or integration of the transplants with host tissue (Schwab, 2002; Kinouchi et al., 2003). Glial scars not only present a physical barrier, but also produce repulsive molecules such as chondroitin sulfate proteoglycans (CSPGs) that inhibit axonal growth (Davies et al., 1996; Fawcett and Asher, 1999; Asher et al., 2001). Several studies have reported increased expression of CSPGs following various transplantation paradigms (Lemons et al., 1999; Plant et al., 2001; Kinouchi et al., 2003; Lakatos et al., 2003), suggesting a possible involvement of CSPGs in the restriction of axonal interaction between transplant and host tissue.

Transplantation of embryonic spinal cord tissue after spinal cord injury (SCI) is a well-established model to examine host axonal growth and functional recovery. After SCI and transplantation into neonates, there is substantial axonal growth not only throughout the transplant, but also within the caudal spinal cord (Bregman, 1987b; Diener and Bregman, 1998a). This anatomical plasticity is accompanied by improved recovery of various motor functions, such as locomotion and skilled reaching (Miya et al., 1997; Diener and Bregman, 1998b). As the animal matures, both the amount of axonal growth and the extent of functional recovery decrease. After injury in adults, fewer descending host axons project into transplant and most of the axons terminate near the hosttransplant interface (Bregman et al., 1997).

In this study, we hypothesized that CSPGs may contribute to the limited extent of axonal growth and functional recovery following transplantation of embryonic spinal cord tissue in adults. Here we show that enzymatic degradation of the inhibitory barrier conferred by CSPGs enhanced transplant-mediated recovery of skilled locomotion and distal forelimb usage and promoted axonal remodeling of host as well as transplanted neurons after SCI in adults.

\section{MATERIALS AND METHODS Animals and surgical procedures}

Adult female Sprague Dawley rats weighing 200-250 g (age 2-3 months; Zivic, Zelienople, PA) were used in this study. They were housed in the Georgetown University Research Resource Facility and all protocols were approved by the Georgetown University Animal Care and Use Committee. Animals received a cervical overhemisection injury at the $\mathrm{C} 4$ level using a procedure modified from that described previously (Bregman et al., 1997). Adult rats were anesthetized with $4 \%$ chloral hydrate (400 $\mathrm{mg} / \mathrm{kg}$ body weight). After laminectomy, the dura was opened and iridectomy scissors were used to create a spinal cord overhemisection. The overhemisection model typically produces a fairly large lesion where the entire right side of the cord plus the left dorsal column and adjacent gray matter are removed, resulting in destruction of more than two-thirds of the transverse area of the cervical spi- nal cord. Vacuum suction was used to aspirate the remaining tissue at the right ventrolateral recess of the canal. For neonatal spinal cord lesions, postnatal day 2 (P2) pups were anesthetized with hypothermia and underwent a surgical overhemisection as previously described (Diener and Bregman, 1998a).

Details of transplant preparation have been described elsewhere (Bregman and McAtee, 1993). Briefly, pregnant female rats were anesthetized at 14 days of gestation. The fetuses were removed individually and maintained in sterile culture medium (Dulbecco's modified Eagle's medium). Fetal spinal cords were dissected and less than 1-mm-long segments of the cord were prepared for transplantation. The transplants were placed into the lesion cavity in direct apposition to the rostral and caudal ends of the lesion. Typically, 3-5 spinal cord segments were transplanted. Gelfoam soaked with enzyme solutions (see below) was placed on top of the dorsal surface of the lesion site or transplants.

\section{Administration of chondroitinase $\mathrm{ABC}$}

Protease-free chondroitinase ABC (C'ase) was purchased from Seikagaku (Japan). A total 0.8 U of C'ase was delivered per animal over the course of the experiment. Immediately after injury and transplantation, $0.2 \mathrm{U}$ of C'ase in $20 \mu \mathrm{L}$ vehicle solution $(0.1 \%$ bovine serum albumin in saline) was applied with gelfoam. The same volume of C'ase solution was administered intrathecally 3, 7, and 11 days after surgery via intrathecal injection. Weight equivalent of penicillinase (P'ase; Sigma, St. Louis, MO) was delivered as a control injection. We injected the enzyme solution into the cisternal space to allow free diffusion to the injury site, less than $1 \mathrm{~cm}$ from the cisterna magna. For intrathecal injection, rats were anesthetized with chloral hydrate and held in an upright position using a stereotaxic apparatus. A small incision was made along the midline above the occipital area and neck muscles were pulled aside with a small retractor to expose the atlantooccipital membrane between the occipital bone and the first cervical vertebra. The membrane was punctured with a $25 \mathrm{G}$ needle tip until the cerebrospinal fluid leaked out. A Hamilton syringe was then introduced to the cisternal space through the hole. Enzyme solution was slowly delivered for 1 minute into the cisternal space and the syringe was pulled out after waiting for 1 more minute. This procedure was subsequently repeated three times at days 3,7 , and 11 . The whole procedure took less than 10 minutes and special care was given to avoid unnecessary muscle damage.

\section{Neuroanatomical tracing}

Anterograde axonal tracing was performed to determine the extent to which injured corticospinal axons regrow into transplant and into the caudal host spinal cord. For anterograde tracing, $5 \mu \mathrm{l}$ of $10 \%$ biotinylated dextran amine (BDA, 10,000 mw; Molecular Probes, Eugene, OR) was pressure-injected into the sensorimotor cortex bilaterally 8 or 9 weeks after injury and 10 days before the animals were sacrificed. FluoroGold (FG; Fluorochrome, Englewood, CO) was used to retrogradely label the cell bodies of transplanted neurons projecting into the host spinal cord in different subsets of animals. A $1-\mathrm{mm}^{3}$ piece of gelfoam pledget was soaked with $1 \mu \mathrm{l}$ of $4 \% \mathrm{FG}$ solution. The gelfoam piece was then divided into 10 smaller pieces and air-dried for 30 minutes. We used a $26 \mathrm{G}$ needle to 
make a small hole at each side of the spinal cord $(\mathrm{C} 8 / \mathrm{T} 1$ level) at the dorsal root entry zone, and then inserted one piece of dry gelfoam pledget into each hole using a finetipped forceps. Leakage of tracer into the cerebrospinal fluid space or through the central canal was minimized using this technique. Rats were sacrificed 4 days later to allow for sufficient transport of the tracer. For each animal, spinal cord sections caudal to the lesion/transplant site (but rostral to the FG tracer site) were analyzed to ensure that tracer had not diffused into or rostral to the transplant. In most animals, the rostrocaudal tracer diffusion was less than one segment.

\section{Immunohistochemistry and quantitative image analysis}

Animals were anesthetized with an overdose of chloral hydrate ( $1 \mathrm{~g} / \mathrm{kg}$ body weight) and perfused with heparinized saline $(0.9 \%)$ followed by $4 \%$ paraformaldehyde in 0.1 $\mathrm{M}$ phosphate buffer, $\mathrm{pH}$ 7.4. The cervical spinal cord was dissected and postfixed in $4 \%$ paraformaldehyde at room temperature for 2 hours, followed by cryoprotection in a graded series of sucrose solutions $(10-30 \%)$ in $0.1 \mathrm{M}$ phosphate buffer at $4^{\circ} \mathrm{C}$. Cryosections of spinal cord $(20 \mu \mathrm{m})$ were cut longitudinally in a 1:6 series and thaw-mounted onto SuperFrost Plus slides (Fisher Scientific, Springfield, $\mathrm{NJ}$ ) and kept at $-20^{\circ} \mathrm{C}$ until use. The rostrocaudal and transverse extent of both the lesion site and transplant apposition were examined by cresyl violet staining and serially reconstructed every $120 \mu \mathrm{m}$ using an aus Jena microprojector and a Zeiss (Oberkochen, Germany) microscope. Animals that received transplants were included in anatomical and behavioral analysis only when transplants were well apposed to the host tissue at least at two of three interfaces (rostral, caudal, and medial).

Longitudinal spinal cord sections were incubated with CS-56 monoclonal antibody (C8035; IgM class; 1:100; Sigma) to determine the expression pattern of CSPGs. This antibody was raised against ventral membranes of the chicken gizzard fibroblasts and recognizes the glycosaminoglycan (GAG) moieties, not core proteins of the CSPGs. Although information on the exact species of GAGs that are recognized by the CS-56 antibody is not available, it has been extensively used as a marker for an increase in overall expression of CSPGs after injury (McKeon et al., 1991; Davies et al., 1999; Camand et al., 2004). 2B6 monoclonal antibody (270432; Seikagaku) was used to examine whether the enzyme was effectively delivered by our method. This antibody was prepared against CSPGs digested with C'ase and specifically recognizes the enzyme-generated epitope on chondroitin 4-sulfate GAG chains (Couchman et al., 1984). 2B6 antibody has been adopted in several previous studies to verify the effective enzyme delivery (Moon et al., 2001; Bradbury et al., 2002; Chau et al., 2004; Fouad et al., 2005). Incubation of a tissue section with C'ase $\left(0.2 \mathrm{U} / \mathrm{ml}, 37^{\circ} \mathrm{C}, 1\right.$ hour), but not $\mathrm{P}^{\prime}$ ase with the same condition, resulted in a dramatic increase in 2B6 immunoreactivity (data not shown), verifying the specificity of the antibody. Polyclonal antibody to the glial fibrillary acidic protein (GFAP) raised in rabbit (AB5804; 1:200 dilution; Chemicon, Temecula, CA) was used to determine the extent of reactive astrogliosis. Staining with this antibody produced immunoreactivity inside and around the transplant very similar to what was reported in our previous studies (Nakamura et al., 2003; Nikulina et al., 2004). Primary antibodies were detected by secondary incubation with Alexa 555 goat antimouse IgM ( $\mu$ chain specific) or IgG (Molecular Probes) for 1 hour at room temperature. To determine the extent of host axonal growth into the transplant, polyclonal anti-serotonin [5-hydroxytryptamine (5-HT)] antibody (20080; ImmunoStar, Hudson, WI) was used at a final dilution of 1:20,000. This rabbit antiserum was generated against serotonin coupled to bovine serum albumin with paraformaldehyde. This antibody visualized only axons with presynaptic bouton-like structures mostly in the gray matter and the pattern of distribution was consistent with the thorough description of the 5-HT immunoreactivity in the previous study (Bregman, 1987a). Peroxidaseantiperoxidase (Sternberger Monoclonals, Lutherville, MD) was used for the development of immunoreactive signals as described elsewhere (Bregman et al., 1997). BDA-traced corticospinal axons were visualized with the Vectastain Elite ABC kit (Vector Laboratories, Burlingame, CA). FG-traced spinal cord tissues were incubated overnight with monoclonal anti-NeuN antibody (MAB377; 1:1,000 dilution; Chemicon), which specifically recognizes the neuron-specific nuclear protein. The specificity of this antibody was tested by staining rat midbrain sections, where only the discrete neuronal population such as rubrospinal neurons was visualized. Reacted primary antibodies were detected by 1-hour incubation with Alexa 555 conjugated goat antimouse secondary antibody (Molecular Probes).

Longitudinal spinal cord sections were analyzed to quantify the serotonin immunoreactive and BDA-traced corticospinal fibers. Growth of the supraspinal axons into transplant did not occur uniformly across the whole transplant. Rather, fiber growth was usually restricted to a small number of sections, and very few axons were found in other areas of transplant even with the C'ase treatment. Therefore, we selected two spinal cord sections separated by at least $120 \mu \mathrm{m}$ that contain the highest amount of axons in the transplant for each animal and obtained the density of axonal fibers inside the transplant. Three nonoverlapped images were photographed with a $10 \times$ objective lens to cover most of the transplant area per each chosen section. The number of pixels occupied by the fibers in the transplant was analyzed using Sigma Scan v. 5.0 (Jandel Scientific, San Rafael, CA) and this number was normalized by the area occupied by transplant to generate the fiber density per unit area. To quantify the serotonergic fibers in the ventral gray matter caudal to the injury site, two longitudinal sections $120 \mu \mathrm{m}$ apart containing ventral gray matter were chosen and the ventral gray matter $3-5 \mathrm{~mm}$ caudal to the distal lesion border was imaged. Since the ventral gray matter showed much higher background reaction than transplant, we needed a higher magnification $(20 \times)$ to accurately discern the immunoreactive axons from background using the image analysis software. The serotonergic fiber density per unit area was obtained in the same way as above. We also obtained the serotonergic fiber density per unit area in the contralateral gray matter at the same level and calculated the percentage of the density of the serotonergic axons in the lesioned side (right) compared to the contralateral side. Since BDA-traced corticospinal fibers in the right side gray matter caudal to the lesion site were very sparsely observed, all the sections on every sixth slide were analyzed to quantify the area occupied by corticospinal axons (typically 18-22 sections per animal). Gray 
matter containing corticospinal fibers ipsilateral to and up to $5 \mathrm{~mm}$ caudal to the lesion was imaged with a $20 \times$ objective lens and the areas occupied by the corticospinal fibers were summed from all the sections per each animal. To adjust the variability in the number of axons that are labeled by BDA, we obtained the density of traced corticospinal fibers coursing along the dorsal columns at the $\mathrm{C} 1 / \mathrm{C} 2$ level in the longitudinal spinal cord sections and calculated the relative ratio for each animal to the mean rostral corticospinal fiber density. This ratio was used to generate a normalized BDA-traced corticospinal fiber density in the transplant and the caudal gray matter. The number of transplanted neurons traced by FG was analyzed in five serial longitudinal sections $240 \mu \mathrm{m}$ apart from each other. We took a representative area at $200 \times$ magnification in each section and manually counted the number of FG-positive neurons. Because the distribution of transplanted neurons is not even throughout the transplant, we normalized the number of FG-positive neurons by that of NeuN-positive neurons in the same area. An unpaired $t$-test and one-way analysis of variance (ANOVA) followed by Tukey's post-hoc analysis were used to compare the measured variables between different experimental groups. Photomicrographs were captured with a Roper Coolsnap fx digital camera (Opelco, Dulles, VA) and Olympus Microsuite software (Olympus Imaging, Melville, NY). Input and out levels were adjusted using Photoshop 7 software (Adobe, San Jose, CA) and images were postprocessed in Photoshop to generate multipanel figures.

\section{Behavioral analysis}

Only the data from the animals that met the following inclusion criteria were included for the final behavioral analysis: 1) animals completed all three behavioral tests (described below); 2) animals had lesions that ablated the dorsal columns bilaterally and lateral and ventral funiculi and intervening gray matter unilaterally (right side), determined by lesion reconstruction; and 3) animals that received transplants had acceptable apposition of transplants to host tissue. All behavioral assessments were performed by an individual who was blind to the kind of enzyme injection (P'ase or C'ase). All test sessions were filmed with a Panasonic SVHS camera (AG-456 Proline) at a shutter speed of 1/1000 second and later analyzed frame by frame in slow motion. A grid walk was used to assess skilled locomotion. The methods for quantitative analysis of locomotor function using a grid runway have been described in detail previously (Kunkel-Bagden et al., 1993). Briefly, rats were trained to cross a grid runway (27 $\mathrm{cm} \times 180 \mathrm{~cm}$ ), with $50 \times 50 \mathrm{~mm}$ holes, for a water reward. Training typically started 3 days after the last enzyme injection. Rats were tested once a week from 3-7 weeks after injury. Each test session consisted of four runs. The average number of limb placement errors per run was obtained from each animal.

Vertical exploration (rearing) and sticker removal tests were used to evaluate forelimb motor function. For the vertical exploration test, rats were placed in a clear, opentopped plastic cylinder measuring $26.5 \mathrm{~cm}$ in height by $17.5 \mathrm{~cm}$ in diameter. In this setting, rats spontaneously rear up and explore a cylinder wall with their forelimbs. The number of wall contacts made by each forelimb individually or together was counted during a 3-minute test session and summed from three test sessions during 5-6 weeks after injury. The percentage of right forelimb wall contacts out of total contacts was determined using the following formula: $[(\mathrm{R}+\mathrm{B}) /(\mathrm{R}+\mathrm{L}+\mathrm{B})] \times 100$ (where $\mathrm{R}=$ number of right forelimb contacts, $\mathrm{L}=$ number of left forelimb contacts, and B = number of both forelimb contacts).

For the sticker removal test, a $1.9-\mathrm{cm}$ diameter sticker was placed on the rat's forehead between the nose and eyes. Rats were freely allowed to remove the sticker during a 3-minute test session. The quality of forelimb movement to remove the sticker was assessed using a ninepoint rating scale adapted from Diener and Bregman (1998b): $0=$ no attempt to remove sticker; $1=$ forelimb did not reach as high as sticker; 2 = forelimb reached as high as the sticker, but moved in lateral plane, unable to adduct functionally towards midline; $3=$ forelimb reached as high as sticker and contacted the head, but no sticker contact occurred; $4=$ forelimb contacted sticker, but sticker was not moved; $5=$ sticker moved, but not fully removed; 6 = sticker fully removed after multiple attempts but without a strong grasp; $7=$ sticker removed after multiple attempts with a strong grasp; $8=$ sticker tightly grasped and swiftly removed. The best score from three test sessions during 5-6 weeks was plotted for each animal. A one-way ANOVA or Kruskal-Wallis test followed by appropriate post-hoc tests were used for statistical comparison of group means at a single timepoint. Repeated measures two-way ANOVA with Tukey's posthoc test was used to compare matched data at multiple timepoints.

\section{RESULTS \\ Expression of CSPGs after overhemisection SCI model and following transplantation}

We examined whether CSPGs increase their expression after the overhemisection spinal injury employed in this study. Although the possibility still exists that core proteins of CSPG molecules are inhibitory to axonal regeneration (Dou and Levine, 1994; Ughrin et al., 2003), several studies have shown that enzymatic degradation of the sulfated chondroitin GAG sidechains was sufficient to overcome an inhibitory influence after various central nervous system (CNS) injuries (Moon et al., 2001; Bradbury et al., 2002; Fouad et al., 2005; Steinmetz et al., 2005). Therefore, we used CS-56 monoclonal antibody that recognizes an epitope on some chondroitin sulfate GAGs to determine a pattern of inhibitory CSPGs expression. We chose a 7-day timepoint because several studies have indicated that abundant CSPGs are observed around the lesions 7-8 days after CNS injury (Asher et al., 2000; Tang X, 2003; Camand et al., 2004). Immunohistochemical staining of longitudinal spinal cord tissue sections showed intense expression of CSPGs along the lesion edges and in scar tissue infiltrating the lesion cavity (Fig. 1A). CSPGs were also deposited in the spinal cord parenchyma, extending more than $100 \mu \mathrm{m}$ from the lesion edges. We also determined whether CSPGs were upregulated following SCI in neonatal rats. In contrast to spinal injury in adults, there was no scar tissue filling the lesion cavity, and CS-56 immunoreactivity in the rostral or caudal cord immediately adjacent to the lesion edges was similar to the basal level at the same 7-day timepoint (Fig. 1B). Therefore, this result indicates that the overhemisec- 

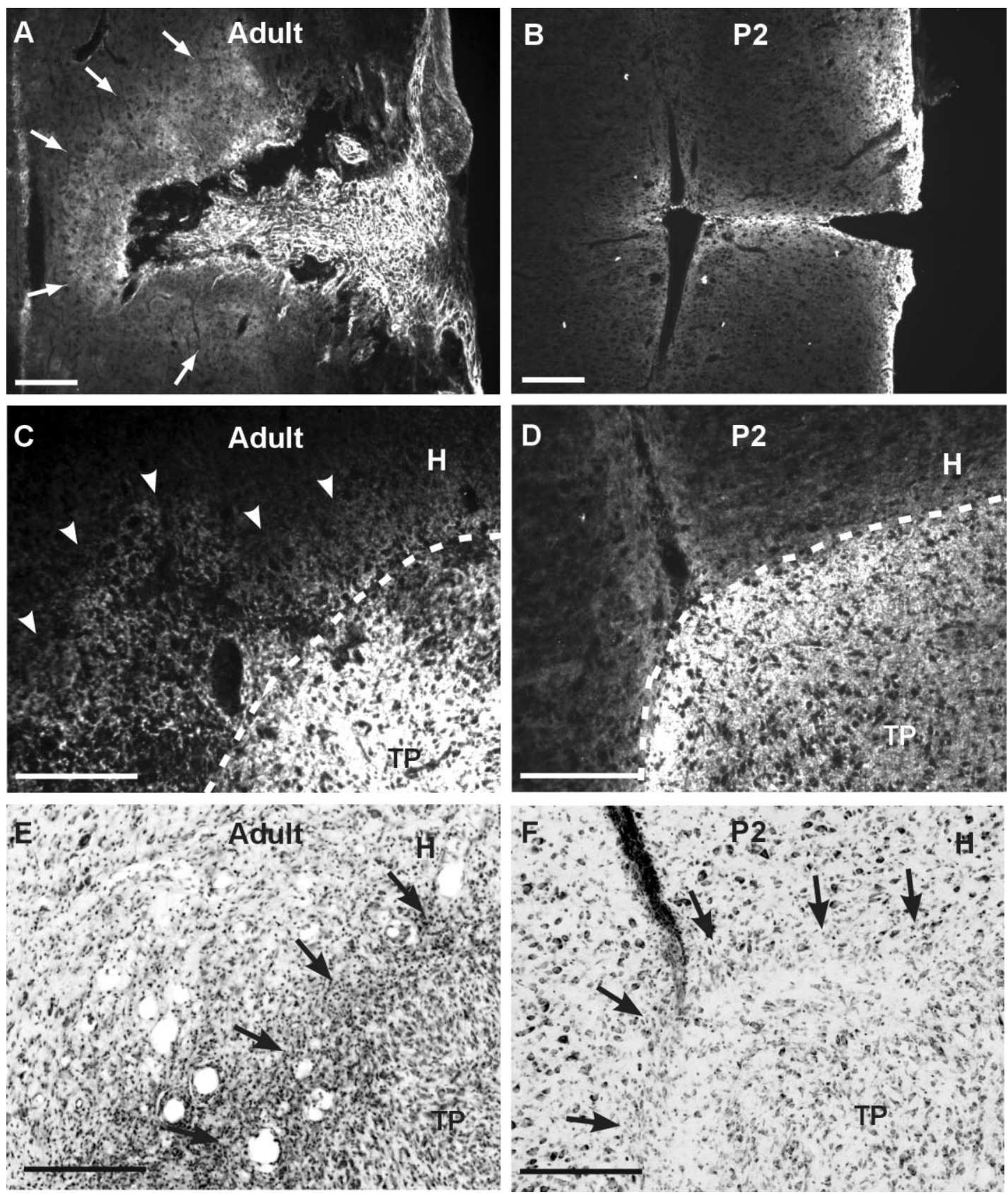

Fig. 1. Expression of chondroitin sulfate proteoglycans (CSPGs) after overhemisection spinal injury in adults and neonates. A,B: Longitudinal spinal cord section was stained with monoclonal CS-56 antibody 7 days after overhemisection injury in adult (A) and neonate 2 days postnatal (P2; B). White arrows in A indicate the extent of CS-56 immunoreactivity in spinal cord around the lesion edges. Rostral is upward and caudal is downward. C,D: CSPGs expression around transplant in adult (C) and P2 (D) host. Spinal cord sections were obtained 14 days after injury and transplantation. Dotted lines indicate putative borders between transplant (TP) and host spinal

tion spinal injury leads to upregulation of CSPGs expression in adult rats, but not in neonates, where embryonic spinal cord grafts lead to substantial anatomical remodeling and enhanced functional recovery compared to adult host (Diener and Bregman, 1998a,b). cord $(\mathrm{H})$, which were determined using cresyl violet staining (see E,F) and/or GFAP immunostaining in adjacent sections. E,F: Cresyl violetstained sections adjacent to the ones in $\mathrm{C}$ and D, respectively. Arrowheads in C indicate the fuzzy CS-56 staining outside of transplant. Black arrows indicate border areas between TP and $\mathrm{H}$ determined on the basis of cytological appearance. The border was more discernable in adult host that that in P2 host, probably due to a massive infiltration of inflammatory cells along the putative border in adults. Scale bars $=100 \mu \mathrm{m}$.

We then examined the expression of CSPGs following transplantation of embryonic spinal cord tissue. Animals were sacrificed 2 weeks after transplantation because it was technically difficult to dissect out lesioned spinal cord with well-apposed transplant earlier than 2 weeks in our 
model. Two weeks after overhemisection injury and transplantation in adult rats, transplanted embryonic spinal cord tissue was surrounded by fuzzy CS-56 immunoreactive signals in the host cord outside of the transplant-host border (Fig. 1C). In contrast, transplants in neonatal hosts were not accompanied by CSPGs deposition in the host tissues, resulting in a distinctive interface between the transplants and host tissues (Fig. 1D). Interestingly, in the adult animals there were numerous small, round inflammatory cells infiltrating the host/transplant borders (Fig. 1E). Inflammatory cells were seldom observed in neonatal hosts (Fig. 1F). Embryonic spinal cord transplants, either in adult or neonatal hosts, showed a very strong CS-56 staining (Fig. 1C,D), which was much more intense than that in the host tissue. The intense CS-56 immunoreactivity was observed throughout the transplant, suggesting that the upregulation of CSPGs in the transplant is probably due to a developmental process rather than spinal injury. Indeed, a recent study reported a high expression of CS-56 immunoreactivity in the intact spinal cord during development (Hsu et al., 2005). We also stained intact spinal cord tissue from embryonic day (E)14 fetus and P7 pups (14 days after E14) and found a similarly strong CS-56 immunoreactivity (data not shown).

In order to verify an effective delivery of C'ase, a subset of animals was sacrificed 3 days after the last C'ase or control P'ase injection. Intrathecal injection of C'ase greatly decreased the CS-56 immunoreactivity that was upregulated after SCI (Fig. 2A,B), demonstrating that C'ase was effectively delivered to the injury site. The extent of fuzzy CS-56 immunoreactivity outside of transplant (Fig. 1C) was reduced after repetitive intrathecal C'ase injection (Fig. 2C). However, the intense CS-56 signals inside the transplant were not altered by C'ase. We also stained the tissue sections with 2B6 antibody, which recognizes an epitope on chondroitin sulfate GAG chains (specifically, chondroitin-4 sulfate) after digestion with C'ase. The 2B6 staining showed intense immunoreactivity along the surface of the spinal cord (data not shown). Interestingly, a sharp immunoreactive band was revealed around the transplant by the 2B6 staining (Fig. 2D). This immunoreactive band was almost completely overlapped with GFAP immunoreactivity formed along the hosttransplant border (Fig. 2E), suggesting that reactive astrocytes deposit a high concentration of CSPGs at the interface between the transplant and host. This 2B6 immunoreactivity along the host-transplant border was not observed in the animals without C'ase injection. Taken together, these results indicate that transplanted embryonic spinal cord tissue in the adult host will be surrounded by CSPGs deposited in the lesioned host cord. The high amount of chondroitin sulfate GAGs at the hosttransplant interface revealed by $2 \mathrm{~B} 6$ staining suggests that a certain interaction between the host tissue and transplant leads to a formation of a molecular barrier that may further inhibit axonal passage into or out of the transplant.

\section{Degradation of CSPGs in combination with transplantation enhances functional recovery}

To determine if CSPGs around the transplant act as a barrier to axonal growth, we degraded the CSPGs using C'ase, which removes chondroitin sulfate GAG chains from the protein core. C'ase was applied at the time of surgery and delivered intrathecally three times postoperatively using the method described in Materials and Methods. Weight equivalent of bacterial enzyme P'ase was used as a control injection.

Data from a total of 56 rats (37, injury plus transplant; 14, injury only; 5 sham operation) were included in the study to determine the effect of C'ase on behavioral recovery and anatomical remodeling. Originally, 45 rats received transplantation of embryonic spinal cord tissue. Of these animals with transplantation, 37 rats (with P'ase, 19; with C'ase, 18) met our inclusion criteria for acceptable transplant (see Materials and Methods), and 8 (with P'ase, 4; with C'ase, 4) rats were excluded entirely from the study due to poor apposition or absence of transplant. The chance of transplantation failure was not different between C'ase- (4/22) and P'ase (4/23)-treated groups. Fourteen rats underwent an overhemisection injury without transplantation (with P'ase, 7; with C'ase, 7). Five sham rats received a cervical laminectomy and the muscle and skin were sutured without damaging the cord. A subset of animals in each group underwent a series of behavioral tests before sacrifice. A total of 29 rats met the inclusion criteria for the behavioral analysis (see Materials and Methods): $\mathrm{n}=6$ for P'ase-only group, $\mathrm{n}=5$ for C'ase-only group, $\mathrm{n}=10$ for transplant (TP) + P'ase group, and $\mathrm{n}=$ 8 for TP + C'ase group.

We examined recovery of locomotion on a grid runway. Walking on the grid runway requires accurate limb placement and thus precise motor control integrated with sensory information on the grid (Kato, 1992; Kunkel-Bagden et al., 1993; Schucht et al., 2002). Sham-operated rats crossed the grid runway with very few errors in limb placement (Fig. 3). Spinal cord injury at C4 resulted in a large number of errors occurring in all four limbs (most severe in the right forelimb), and C'ase injection alone without transplantation failed to induce any improvement. Transplantation with control injections slightly decreased the mean number of errors and C'ase treatment following transplantation further improved accuracy in limb placement. Repeated measures two-way ANOVA showed significant group effects between different treatment groups $(F=6.36 ; P<0.01)$. Significant difference by Tukey's post-hoc analysis was found only in the transplant plus C'ase group compared to P'ase- or C'ase-only group $(P<0.01)$. Univariate ANOVA at each timepoint indicated that the significant group effect became evident by 4 weeks after injury and persisted until 7 weeks, the last timepoint measured $(P<0.05$ at 4 and 5 weeks; $P<$ 0.01 at 6 and 7 weeks).

Spinal cord injury rostral to the cervical enlargement results in substantial deficits in forelimb motor function (Schrimsher and Reier, 1993). After a right overhemisection injury at the C4 level severe impairment of motor function develops in the right forelimb. Motor function of the right forelimb was assessed at 5 and 6 weeks after injury and the extent of recovery was compared between different experimental groups. Asymmetry of forelimb use was measured during vertical exploration in a plastic cylinder. Sham-operated animals used both forelimbs without any preference when they explored the cylinder wall. This symmetric forelimb use resulted in $70 \%$ right forelimb contacts out of total wall contacts (Fig. 4A), because we counted the contacts with both right forelimb only and bilateral forelimbs as right forelimb contacts (see Materi- 

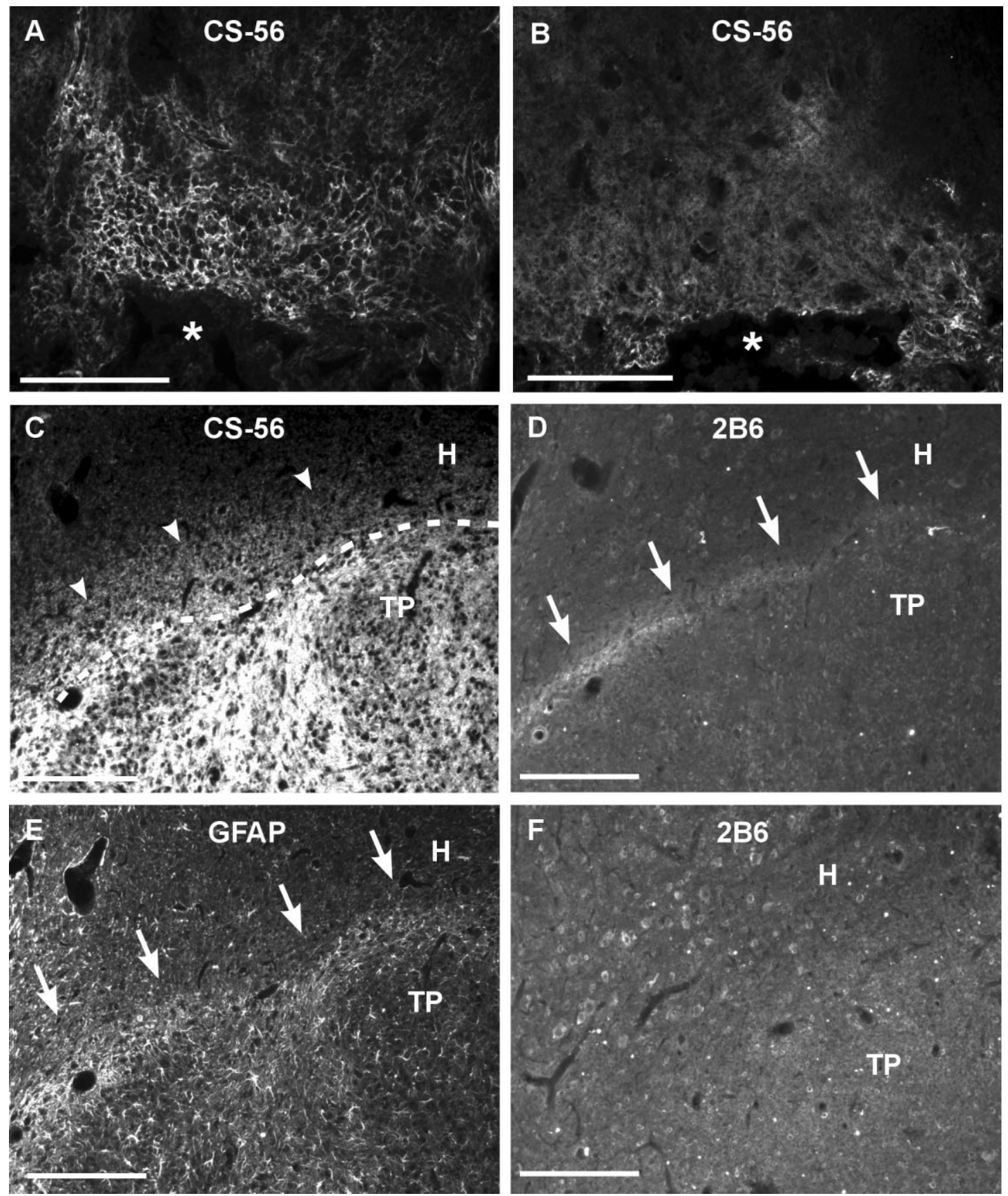

Fig. 2. Verification of effective delivery of chondroitinase $A B C$ (C'ase). A,B: CS-56 immunostaining in the lesioned spinal cord sections. Animals were injected either with C'ase (B) or control enzyme penicillinase (P'ase; A) three times ( 3,7 , and 11 days after injury) and sacrificed 14 days after spinal injury. The intensity of CS-56 staining was greatly reduced after C'ase treatment (B). Asterisk indicates lesion cavity. C: CS-56 immunostaining in the spinal cord sections 14 days after injury and transplantation. C'ase was injected with the same schedule as above. A dotted line indicates a border between

als and Methods). After the right-sided overhemisection injury at $\mathrm{C} 4$, the percentage of right forelimb contacts dropped to $30 \%$ as injured animals preferentially used the left forelimbs to contact the cylinder wall. Injection of transplant (TP) and host spinal cord $(\mathrm{H})$. Arrowheads indicate the extent of CS-56 immunoreactivity outside of transplant. D: 2B6 immunostaining of section adjacent to the one in C. White arrows indicate 2B6-positive immunoreactive band along the TP-H interface. E: GFAP staining of spinal cord section adjacent to the one in $\mathrm{C}$ and D. GFAP immunoreactivity indicated by white arrows was overlapped with 2 B6 immunoreactivity shown in D. F: 2 B6 staining in the rat without C'ase. Without C'ase, there was no discernable border between host and transplant with 2B6 staining. Scale bars $=100 \mu \mathrm{m}$.

C'ase did not improve right forelimb use compared to control P'ase injection. Transplantation of embryonic spinal cord tissue did not increase the percentage of right forelimb contacts regardless of P'ase or C'ase injection. 


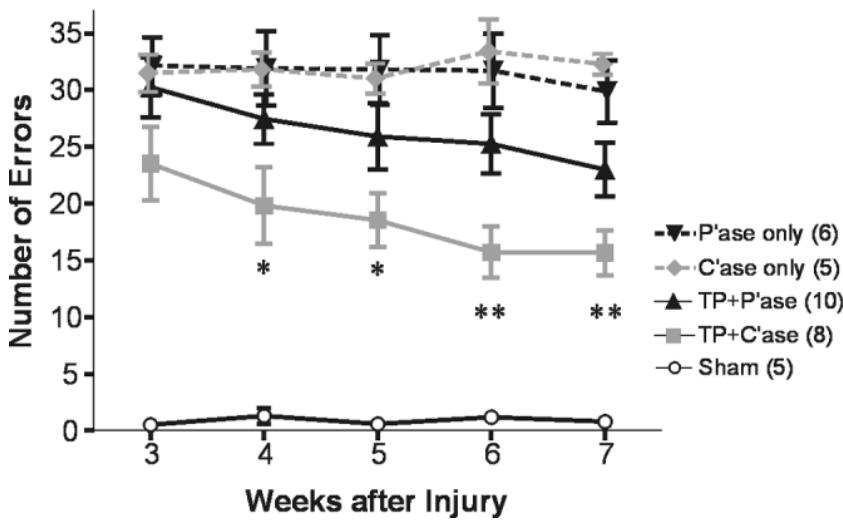

Fig. 3. Recovery of locomotion on a grid runway. Skilled locomotion on a grid runway was tested weekly from 3-7 weeks after injury. Average number of limb placement errors per run was obtained from each animal and group means \pm SEM are plotted for the indicated timepoints. Although chondroitinase ABC (C'ase) injection alone did not improve the accuracy of grid walk, C'ase injection combined with transplant (TP) significantly decrease the number of errors from 4 weeks after injury and thereafter. $* P<0.05 ; * * P<0.01$ compared to penicillinase (P'ase) or C'ase only group (one-way ANOVA with Tukey's post hoc).

Instead, we observed qualitative difference in distal forelimb movement when animals tried to contact the cylinder wall with their right forelimbs. When the injured right forelimb was used to contact the wall, animals frequently showed a lack of distal control of the injured forelimb by placing the dorsum of their paw against the wall instead of the expected palmar side (Fig. 4B). This abnormal forelimb movement was never observed in sham-operated control rats. We defined the right forelimb contact with the wrist joint extended more than $180^{\circ}$ (dorsiflexion) as a functional contact (normal behavior), and obtained the percentage of the functional contacts out of the total right forelimbs contacts (right forelimb only + bilateral forelimbs). We excluded the data from animals whose number of total right contacts was less than five. As a result, one rat from C'ase-only and one from TP + P'ase group were not included. Cervical overhemisection injury resulted in a decrease in the frequency of functional contacts (Fig. $4 \mathrm{C})$. C'ase injection without transplant failed to produce any significant difference. Transplantation with control P'ase injection showed an increase in the frequency of functional contacts, indicating that the transplant improved forelimb function. This recovery was improved even further with C'ase. In all TP + C'ase rats, more than $75 \%$ of the right forelimb contacts were functional. The Kruskal-Wallis test revealed a significant difference between groups $(P<0.01)$ and Dunn's post-hoc analysis showed that the significant difference existed between TP plus C'ase group and control P'ase- or C'ase-only group $(P<0.05$ and $P<0.01$, respectively). This result suggests that although the recovery of symmetric forelimb use during vertical exploration is not affected, removing the inhibitory properties of CSPGs following transplantation does lead to improved control of distal forelimb movement.

A sticker removal test was used to examine recovery of skilled reaching behavior of the right forelimb (Diener and Bregman, 1998b). Sham-operated control rats swiftly grasped and removed the sticker from the head using either or both forelimbs. After a right overhemisection injury, animals almost always used the left forelimb to remove the sticker. Attempts with the right forelimb to reach for the sticker, although unsuccessful, were still observed in most test sessions, either independently or assisting the left forelimb. Qualitative differences in the usage of the right forelimb during these attempts were determined using one of the rating scores ranging from 0-8 according to the scale described by Diener and Bregman (1998b). After the injury, animals were not able to move their right forelimb to the sticker (all scores less than 3) irrespective of control P'ase or C'ase injection (Fig. 4D). Transplantation appeared to partially improve the quality of right forelimb usage. Again, only rats treated with transplant plus C'ase injection showed statistically significant improvement in the quality of right forelimb usage in this test compared to the P'ase- or C'ase-only group $(P<0.05$ and $P<0.01$, respectively, by KruskalWallis with Dunn's post hoc). Taken together, our results in behavioral analysis showed that removal of the inhibitory properties of CSPGs increased the extent of functional recovery following transplantation, suggesting that deposition of CSPGs limits the extent of transplantmediated functional recovery in the adult.

\section{Raphespinal axon remodeling is promoted by C'ase treatment following transplantation}

To address whether enzymatic degradation of CSPGs allows more axonal growth into transplant, raphespinal axon growth in the transplant was visualized by serotonin immunostaining. Raphespinal axons reach the spinal cord after E14 (Bregman, 1987a), thus any serotoninimmunoreactive fibers found in the transplant would result from regenerating or sprouting axons of the host raphespinal axons. A greater amount of serotonin immunoreactive fibers within transplant was observed in rats treated with C'ase than those with control P'ase injection 8 or 9 weeks after injury and transplantation (Fig. 5A,B). Serotonergic fibers were observed more frequently near the host-transplant interface, but a large amount of axon growth sometimes could be observed even at the central area of transplant in the rats that received C'ase following transplantation. Quantification of the density of serotonin-immunoreactive fibers in transplant showed a significant increase following C'ase injection (Fig. 5C; $P<$ 0.05 by unpaired $t$-test).

We compared the extent of reinnervation of the caudal spinal cord by the raphespinal axons between different experimental groups. The percentage of the density per unit area of the serotonergic fibers in the right (injured) compared to the left side was obtained at the ventral gray matter 3-5 $\mathrm{mm}$ caudal to the distal lesion border, which corresponds to the C6/7 spinal cord segment. After an overhemisection injury the density of serotonin immunoreactive fibers was reduced to $21.7 \%$ of contralateral gray matter (Fig. 6A). C'ase injection only and transplant with control P'ase injection slightly increase the mean percentage of serotonin fibers $(26.3 \%$ and $30.4 \%$, respectively). C'ase injection combined with transplant further increased the extent of serotonergic innervation up to $45.2 \%$ of the contralateral side (Fig. 6B-E). Statistical analysis with one-way ANOVA revealed a significant group difference $(F=4.552 ; P<0.01)$ and Tukey's post-hoc analysis 


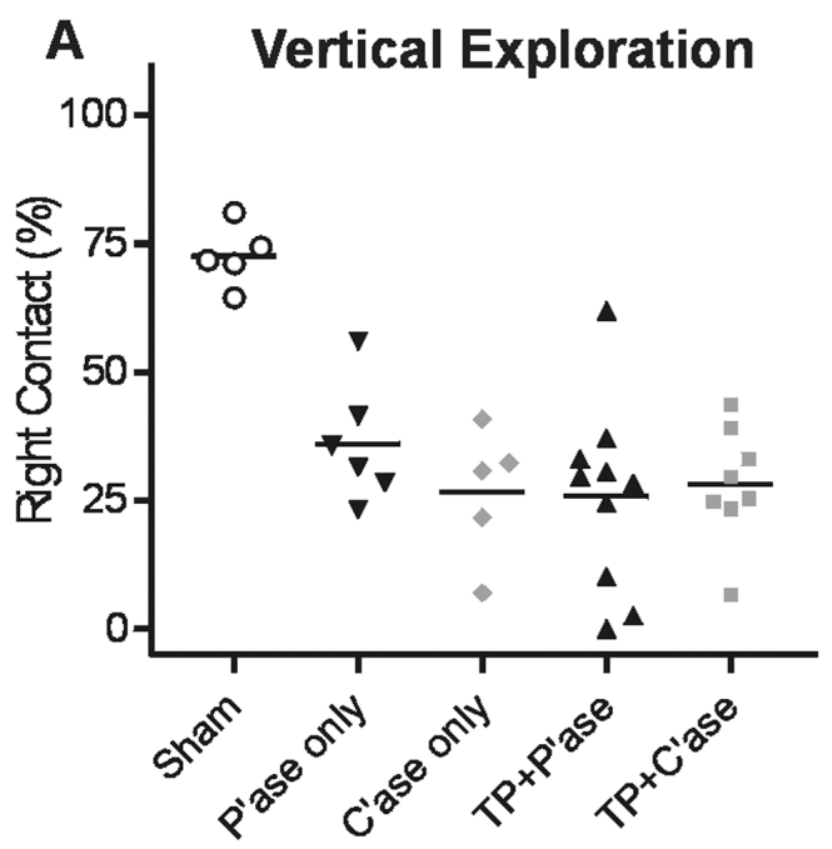

B
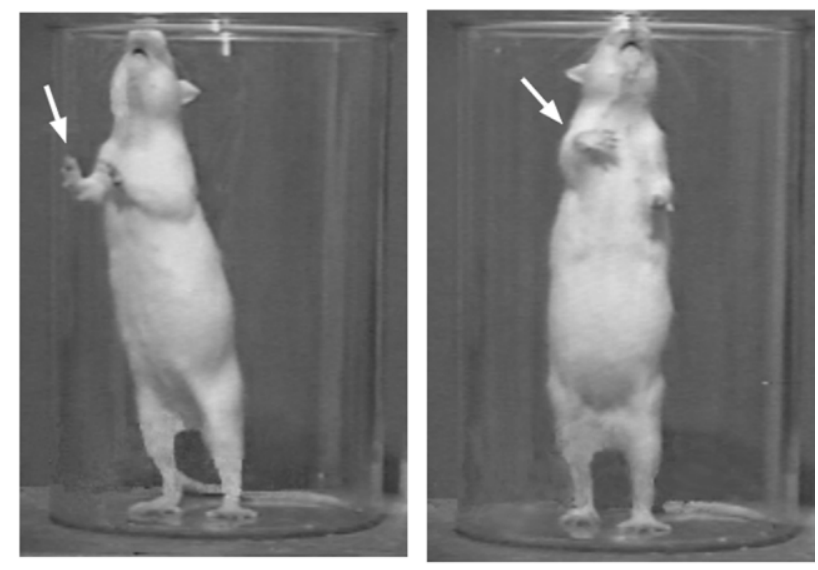

C Vertical Exploration

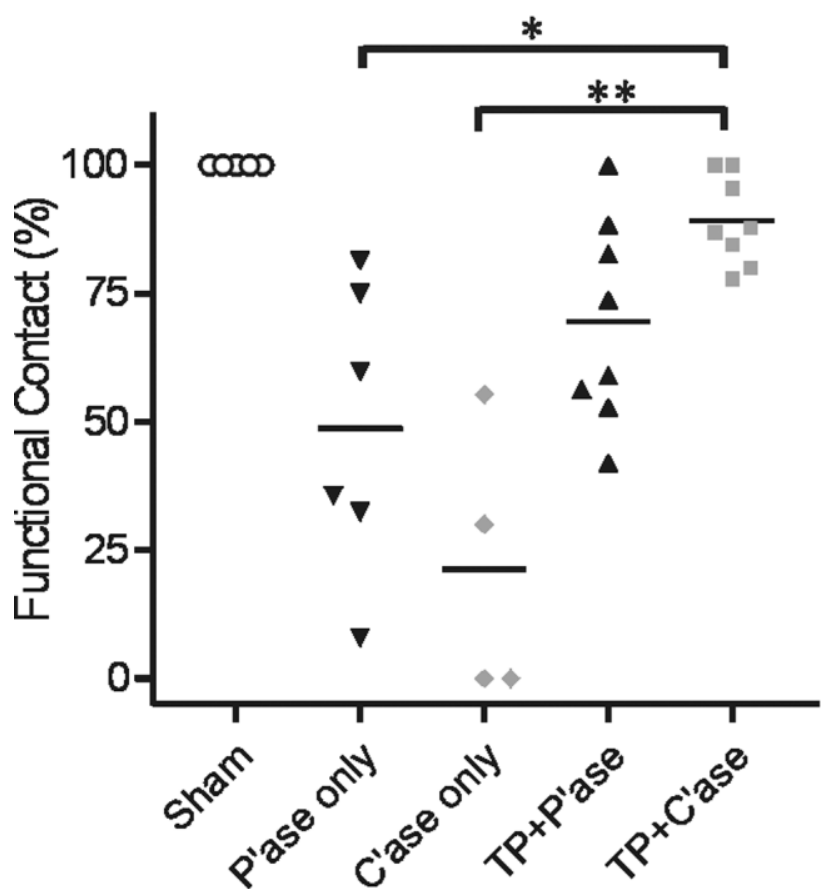

Fig. 4. Recovery of forelimb motor function. All forelimb motor function tests were performed at 5 or 6 weeks after injury. A: Percentage of right forelimb contacts to the cylinder wall during vertical exploration test. There was no significant difference between different treatment groups. B: Illustration of qualitative difference in distal forelimb usage during vertical exploration behavior. Uninjured rats always contact the wall with the palm side of their forepaws (arrow, left panel). Injured rats frequently place the dorsum side of their paw

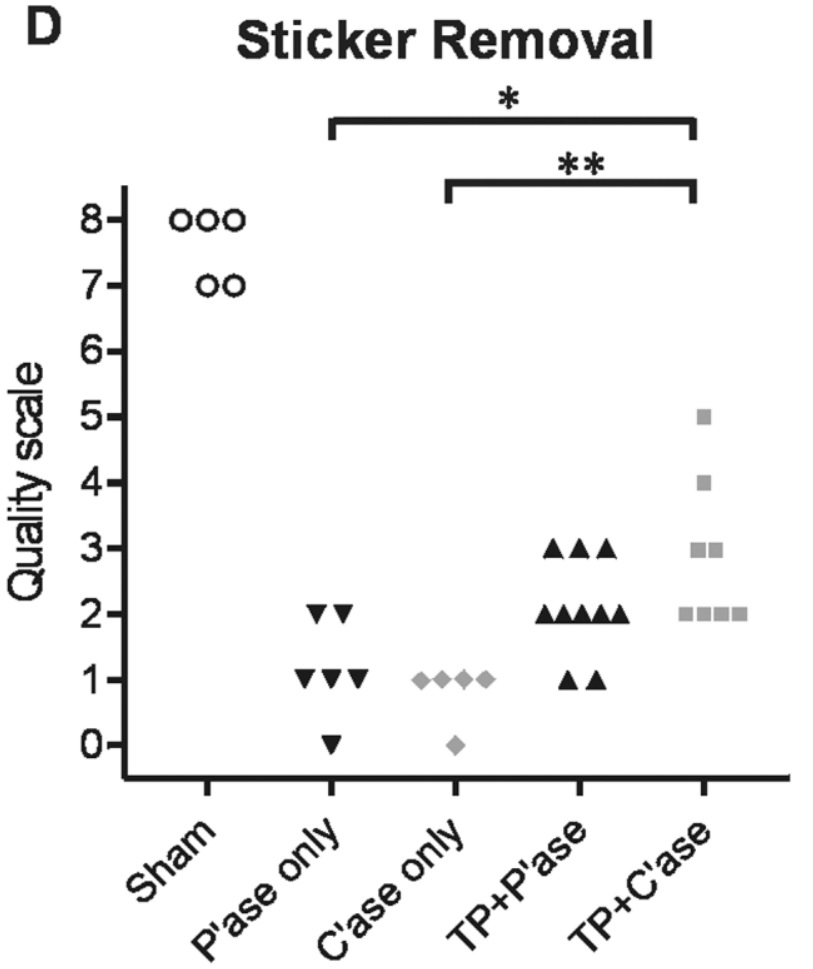

against the cylinder wall (arrow, right panel). C: Quantification of functional contacts defined as the ones with the wrist joint extended more than $180^{\circ}$ (dorsiflexion). Each data point represents the percentage of functional contacts out of total right forelimb wall contacts. D: Sticker removal test. Each data point represents the best scale from three test sessions of each animal. TP = transplant. C,D: $* P<$ 0.05 compared to P'ase-only group (Kruskal-Wallis test with Dunn's post hoc). 


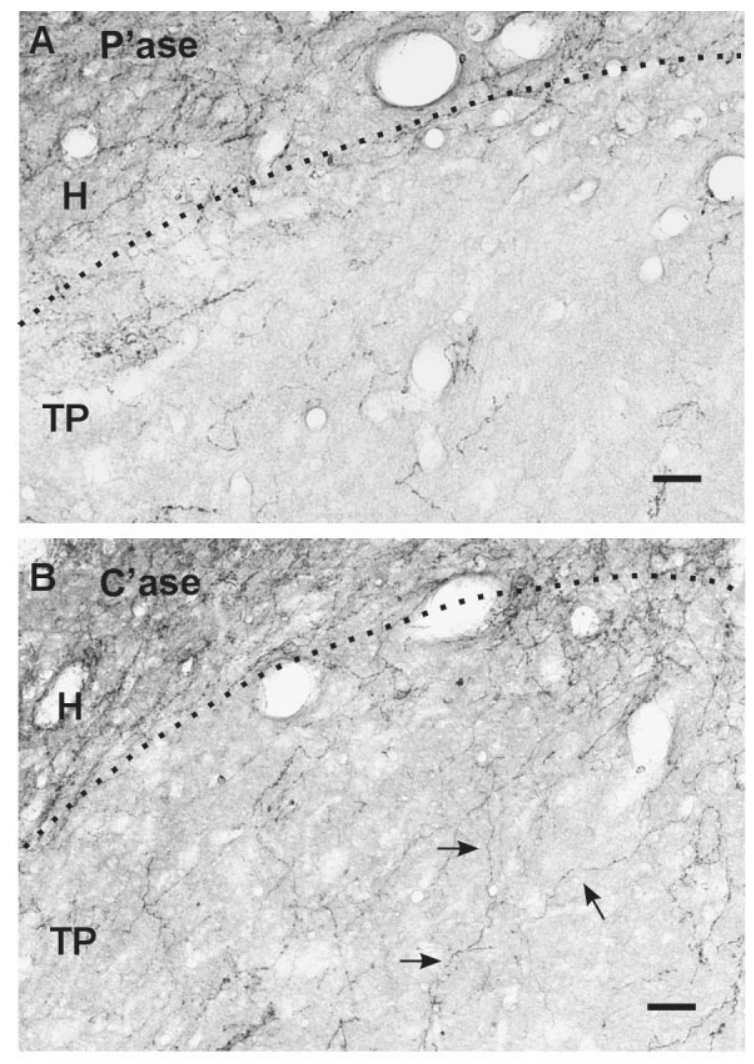

C

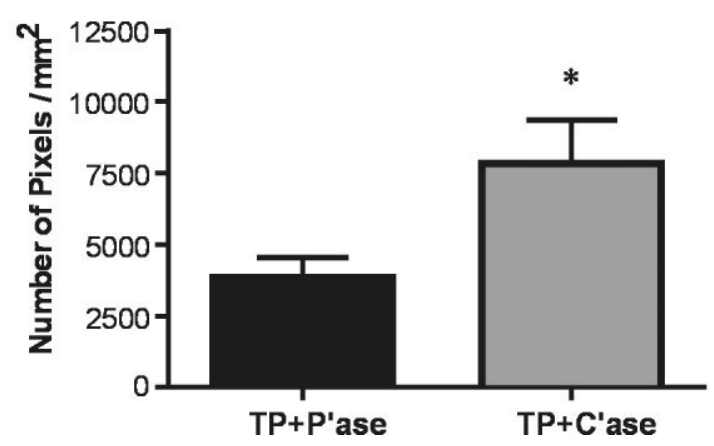

Fig. 5. Serotonergic axon growth into transplant. A,B: Representative images of the longitudinal spinal cord sections stained with anti-serotonin antibody. Dotted lines indicate the interface between host $(\mathrm{H})$ and transplant (TP). The amount of serotonergic fiber was higher inside the transplant after chondroitinase ABC (C'ase) treatment (B) compared to control penicillinase (P'ase) injection (A). Moreover, lengthy growth of serotonin fiber (arrows) was more frequently observed following C'ase treatment. Quantification of serotonergic fiber density per $1 \mathrm{~mm}^{2}$ of the transplant showed a significant increase by C'ase treatment. Data are presented as group mean \pm SEM $\mathrm{n}=13$ for $\mathrm{TP}+\mathrm{P}$ 'ase and $\mathrm{n}=12$ for $\mathrm{TP}+$ C'ase group. $* P<0.05$ (by unpaired $t$-test). Scale bar $=100 \mu \mathrm{m}$ in A,B.

showed the only significant difference between control (P'ase-only) and TP plus C'ase group $(P<0.05)$. This result was not affected by the variability in the amount of serotonergic fibers in the contralateral gray matter because the serotonin fiber density in the left side was not significantly different between all the groups $(P=0.59$;
P'ase-only, 13,174 \pm 885; C'ase-only, 14,069 \pm 1,745; TP + P'ase, $16,800 \pm 2,311$; TP + C'ase, 15,622 $\pm 1,540$ ). Together, these data show that enzymatic degradation of CSPGs deposited around the transplant increases the raphespinal axon growth into the transplant, and C'ase treatment in combination with transplant significantly increases the extent of raphespinal reinnervation of the cervical motor region caudal to the injury site.

\section{Remodeling of corticospinal axons is not affected by degradation of CSPGs}

To examine the extent of remodeling of the corticospinal axons, we used anterograde axonal tracing with BDA. In the rats without transplant, corticospinal axons in the dorsal columns stopped abruptly at the injury site, forming retraction bulbs (Fig. 7A), and did not show any appreciable evidence of regeneration beyond the injury site with or without C'ase. With the lesioned cavity bridged by transplant, corticospinal axons grew into the transplant (Fig. 7B). Typically, corticospinal axon growth into the transplant was spatially restricted to the area close to the rostral interface between transplant and host tissue in both P'ase- and C'ase-treated rats. Quantification of the density per unit area of the corticospinal axons in transplant revealed no significant difference between the two groups; mean pixel number of corticospinal axons per 1 $\mathrm{mm}^{2}$ of transplant was $1,111 \pm 412$ in TP plus P'ase group $(\mathrm{n}=6)$ and 1,737 \pm 752 in TP plus C'ase-treated rats $(\mathrm{n}=$ 6 ). At the spinal cord caudal to the injury site, we could not find any BDA-traced fibers in the white matter tracts of the dorsal columns even when the lesion cavity was bridged by transplant. C'ase injection did not affect this observation. Corticospinal axons innervating the right side cervical cord caudal to the injury were rarely observed in the gray matter after an overhemisection injury (Fig. 7C). Most of the observed axons were found in the dorsal half of the gray matter and they seemed to be more frequently located close to the midline, suggesting that they might be crossed terminations of the spared left dorsolateral corticospinal tract. To determine whether corticospinal innervation to the right gray matter caudal to the injury site was promoted by any treatment, we counted the BDA-traced fibers in the gray matter up to 5 $\mathrm{mm}$ caudal to the injury site. Normalized areas occupied by corticospinal axons were not significantly different across the different groups: 2,758 $\pm 853 \mu \mathrm{m}^{2}$ in P'ase-only group $(\mathrm{n}=7), 2,571 \pm 1,232 \mu \mathrm{m}^{2}$ in C'ase-only group $(\mathrm{n}=$ $7), 2,650 \pm 1,392 \mu \mathrm{m}^{2}$ in TP plus P'ase group $(\mathrm{n}=6)$, $2,164 \pm 751 \mu \mathrm{m}^{2}$ in TP plus C'ase group $(\mathrm{n}=6)$. These data show that, in contrast to serotonergic raphespinal axons, remodeling of corticospinal axons was not affected by C'ase treatment even when combined with transplantation.

\section{Degradation of CSPGs supports axonal growth of transplanted neurons to the host spinal cord}

We have proposed that transplanted neurons can relay disconnected supraspinal or propriospinal inputs to the spinal motor networks and this may be an underlying mechanism for transplant-mediated functional recovery in adult SCI (Bregman et al., 1993). Anterograde and retrograde neuroanatomical tracing indeed showed that transplanted embryonic neurons can extend their axons 

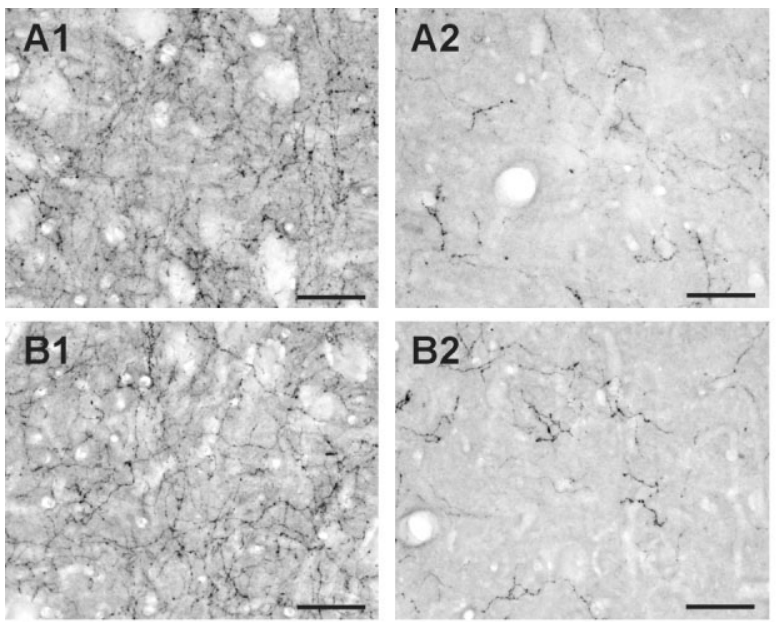

$E$

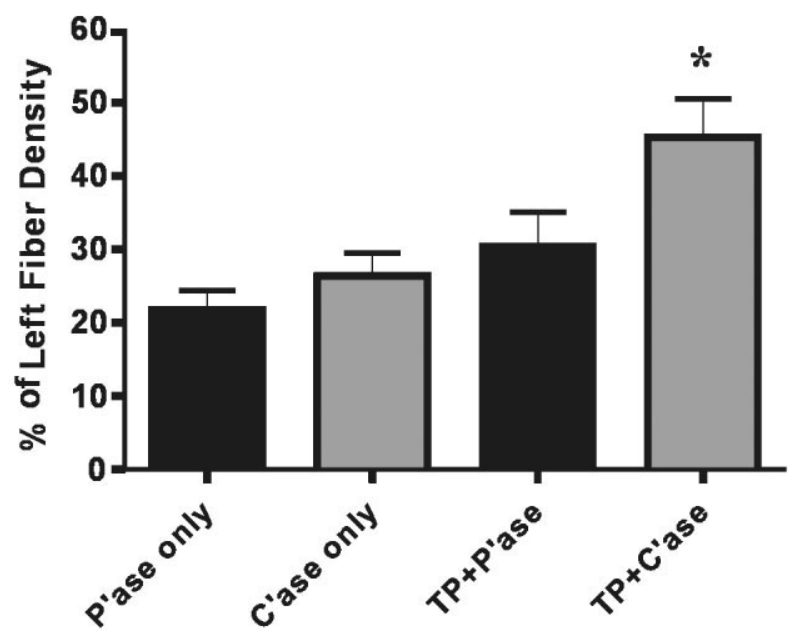

Fig. 6. Serotonergic fiber innervation to the ventral gray matter caudal to the injury site. A-D: Representative images of the caudal ventral gray matter. A: Penicillinase only (P'ase). B: Chondroitinase ABC (C'ase) only. C: Transplant (TP) + P'ase. D: TP + C'ase. After a right-sided overhemisection injury, the density of serotonin immunoreactive fibers in the right side (right column of each pair, designated as number 2) is dramatically reduced compared to that of contralateral ventral gray matter at the same segmental level (left column of

as far as several millimeters from the host-transplant interface in adult rats (Jakeman and Reier, 1991). When transplanted into the cervical injury site in neonates, they established more frequent axonal connections with host tissue, and even long connections with the lumbar spinal cord could be observed (Reier et al., 1986; Diener and Bregman, 1998a). We reasoned that the difference in axonal growth of transplanted neurons between neonatal and adult hosts can be explained by the inhospitable environment of the mature CNS. Here we tested whether degradation of CSPGs can support axonal growth from the transplanted neurons to host cervical spinal cord in adult.

We used a retrograde labeling technique to identify transplanted neurons that extend axons into the host cervical enlargement caudal to injury where the motor neurons for
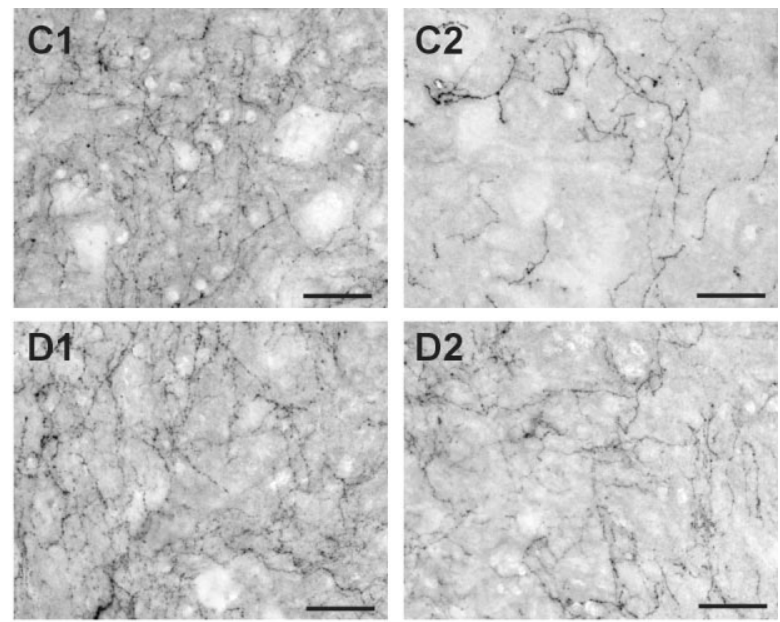

each pair, designated as number 1). Most robust regrowth was observed in TP + C'ase group (D). Quantification of the percentage of serotonin fiber density in the right side compared to the left $(\mathbf{E})$. TP + C'ase group showed significant difference compared to P'ase only group. Data are presented as group mean \pm SEM. $\mathrm{n}=7$ for P'aseonly, $\mathrm{n}=7$ for C'ase-only, $\mathrm{n}=13$ for TP + P'ase, and $\mathrm{n}=12$ for TP + C'ase group. ${ }^{*} P<0.05$ (one-way ANOVA with Tukey's post hoc). Scale bar $=100 \mu \mathrm{m}$ in A-D.

forelimb movement are located. To avoid a possible passive diffusion, small dried pieces of FluoroGold (FG)-soaked gelfoam were inserted into the bilateral cervical cord at the $\mathrm{C} 8 / \mathrm{T} 1$ level, which is far below the C4 lesion site. After harvesting the spinal cord tissue we measured the actual distance from the center of the transplant to the injection sites and included the cases only with the actual distance of at least $8 \mathrm{~mm}$. The interface between the host gray matter and transplanted tissue was easily identifiable due to an abrupt decrease in the density of labeled neurons between adjoining host and transplanted tissue, suggesting that FG fluorescence in the transplant was not due to a passive diffusion from the injection site. Fluorescent intensity of the transplanted neurons, if observed, was generally lower than that of ubiquitous propriospinal neurons in the host gray 

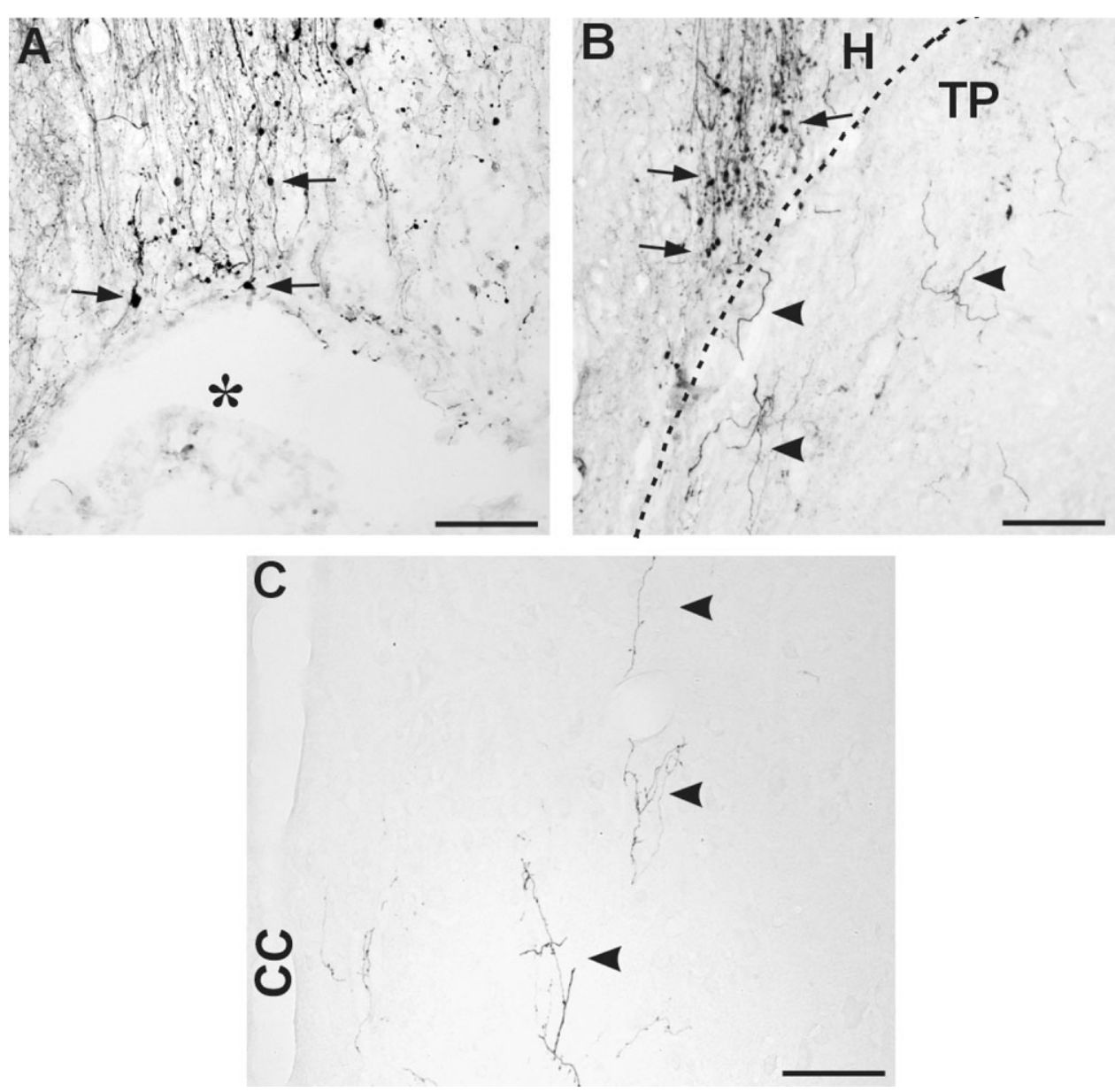

Fig. 7. Corticospinal axon growth. A,B: Representative images of the longitudinal spinal cord sections from the rats with injury only (A) and following transplantation (B). A: Following spinal cord injury, biotinylated dextran amine (BDA)-traced corticospinal fibers abruptly stop at the injury site, forming retraction bulbs (arrows). There was no evidence of corticospinal axon regeneration beyond the injury site through spared spinal cord tissue with or without chondroitinase $A B C$ (C'ase). Asterisk indicates the lesion cavity. B: When the cavity was bridged by transplant (TP), corticospinal axons start to grow into TP (arrowheads). The amount of fiber growth, however, was not significantly increased by C'ase treatment. Dotted line indicates the interface between host $(\mathrm{H})$ and TP. Rostral is upward and caudal is downward. C: Very few, if any, corticospinal axons were observed in the host spinal cord (right) caudal to the injury site (arrowheads). None of the treatments improved the growth of the injured corticospinal axons in the caudal spinal cord. $\mathrm{CC}=$ central canal. Scale bar $=100 \mu \mathrm{m}$ matter. FG positive neurons were counted only when they had clear neuronal morphology and contained mature neuronal nuclear protein NeuN as revealed by immunolabeling of the same section (Fig. 8A,D). Transplanted neurons that were traced by FG were rarely observed in the transplant of all the rats with control P'ase injection (Fig. 8B). In C'asetreated rats, FG-positive neurons were much more frequently observed (Fig. 8E). Quantification of FG-positive neurons per 1,000 transplanted neurons revealed that removal of inhibitory properties of CSPGs significantly increased the number of FG-positive neurons by more than 4 -fold (Fig. 8G; $P<0.05$ by unpaired $t$-test). This result suggests that CSPGs deposition restricts not only axonal growth of the host neurons but also axonal growth from the transplanted neurons into the host spinal cord.

\section{DISCUSSION}

Previous work from our laboratory has shown that the extent of anatomical remodeling and functional recovery mediated by embryonic spinal cord transplants is robust in neonates, but declines as the animal matures. In this study we show that degradation of CSPGs promotes axonal interaction between host and transplant and enhances functional recovery following transplantation in adult rats. This suggests that CSPGs deposited around the transplant constitute a molecular barrier to axonal passage and may be at least in part responsible for the restriction of transplant-mediated axonal remodeling and functional recovery in adults. We believe that developmental changes in intrinsic neuronal factors also significantly contribute to this decline (Bregman et al., 1989; Cai et al., 2001). Our results suggest that removal of the inhibitory molecular barrier increases the extent of transplant-mediated axonal remodeling and functional recovery in the mature CNS, where the neuronal capacity to regrow is already diminished.

Disruption of axonal connections between the supraspinal neurons and spinal motor circuits results in devastating functional deficits after SCI. Various transplantation 

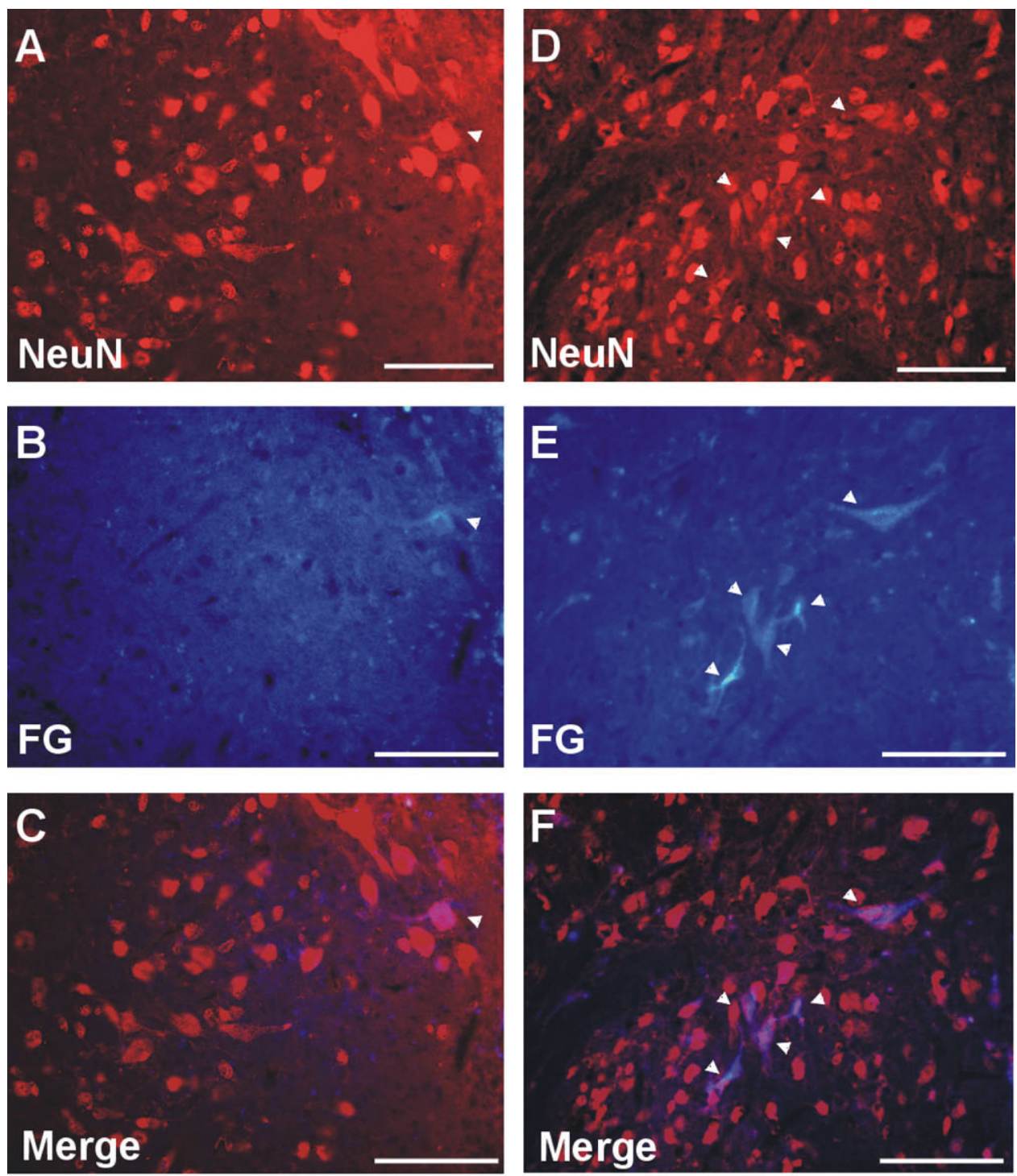

G

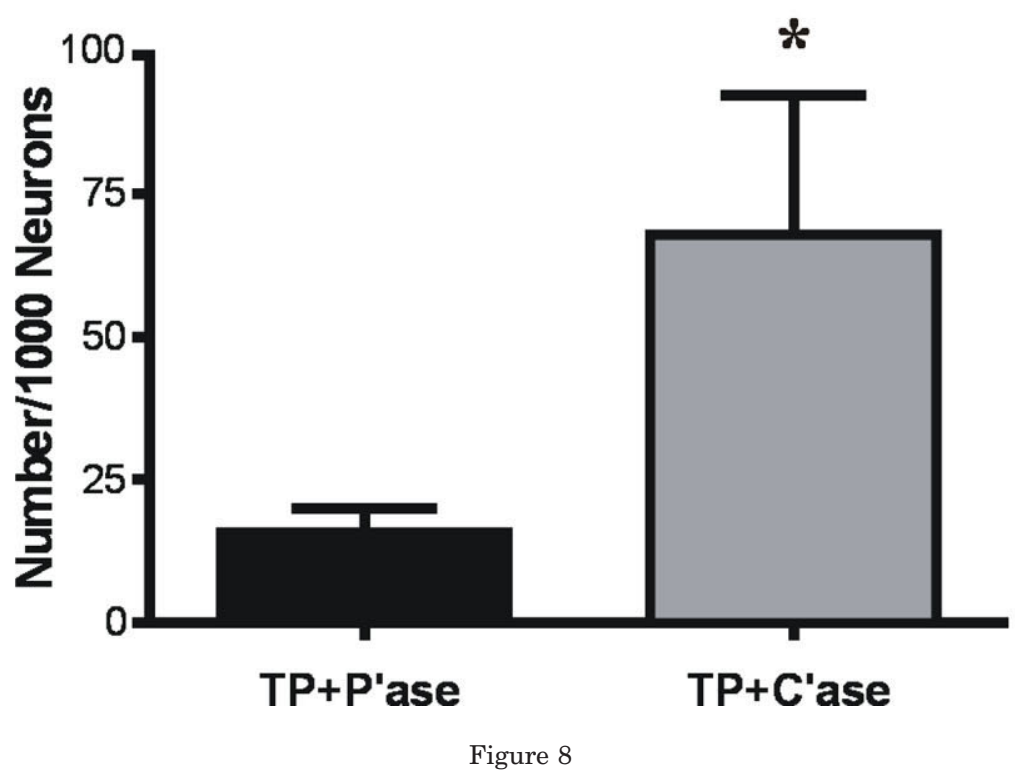


strategies have been employed to restore the severed connections in experimental SCI, leading to a variable degree of functional improvement (Cheng et al., 1996; Diener and Bregman, 1998b; Liu et al., 1999; Ramon-Cueto et al., 2000; Keyvan-Fouladi et al., 2003). Transplanted cells or tissues will function as growth-permissive scaffolds for regenerating axons to grow beyond the injury site. In addition, cellular transplantation has the potential to replenish lost neurons and/or glial cells (McDonald et al., 1999). A glial response and resultant CSPGs production may be unavoidable in these transplantation strategies because traumatic injury leads to a deposition of various species of CSPGs around the lesioned spinal cord (Lemons et al., 1999; Jones et al., 2003a,b; Camand et al., 2004). Furthermore, implanted cells or tissues recognized as foreign by the host immune system will induce reactive astrocytosis and proliferation of other immune cells such as macrophages that can produce CSPGs (Fitch and Silver, 1997; Jones et al., 2002). Indeed, increased deposition of CSPGs has been reported after transplantation of Schwann cells (Plant et al., 2001; Lakatos et al., 2003), olfactory ensheathing glial cells (Lakatos et al., 2003), retinal transplants (Kinouchi et al., 2003), and embryonic spinal cord tissues (Lemons et al., 1999). An inhibitory barrier conferred by upregulation of CSPGs may reduce the capacity of transplants to guide growing axons or prevent proper integration of transplanted cells. Indeed, a recent study reported that infusion of C'ase enhanced axonal regrowth to the caudal host spinal cord through Schwann cell-seeded guidance channels (Chau et al., 2004). Our study further shows that degradation of CSPGs promotes axonal growth of transplanted neurons as well and, more important, increases the extent of functional restoration mediated by transplantation.

Expression of CSPGs increased around the lesion cavity in adult rats but not in neonates. This is consistent with the finding that glial scar explants from neonates contain very little CSPGs and support rather than inhibit axonal growth (McKeon et al., 1991). The lack of CSPGs upregulation in neonates may be related to a weaker astroglial reaction than that observed in adults (Barrett et al., 1984; Firkins et al., 1993). Embryonic spinal cord transplants into adult hosts, therefore, will be exposed to growthinhibitory CSPGs deposited in the injured host cord. In contrast, transplants in neonatal operates will be integrated to host tissue without being exposed to injuryinduced CSPGs and may provide a favorable environment to the passing axons through the host-transplant border.

Fig. 8. Chondroitinase ABC (C'ase) supports growth of axons from transplanted neurons to the host spinal cord. A-C: Transplant (TP) plus penicillinase (P'ase) group. D-F: TP plus C'ase group. A,D: Longitudinal sections of the transplant (TP) were stained with mature neuronal marker NeuN 9 weeks after injury and transplantation. B,E: Neurons in TP that grew axons to the host spinal cord were identified by retrograde tracing using FluoroGold (FG)-injected into the C8/T1 segment. C,F: The number of FG-positive cells with NeuN immunoreactivity in merged image was counted for quantification. Arrowheads indicate the same neurons that are doubly positive for FG and NeuN. G: Quantification of the number of FG-positive neurons normalized by the total number of transplanted neurons (NeuNpositive). Data are presented as group mean \pm SEM. $\mathrm{n}=7$ for TP + P'ase, $\mathrm{n}=5$ for TP + C'ase group. $* P<0.05$ (unpaired $t$-test). Scale bar $=100 \mu \mathrm{m}$.
We found intense CS-56 immunoreactivity in transplant regardless of host age. Developing spinal cord (without injury) contains a high amount of CS-56 immunoreactivity (Hsu et al., 2005) and time-matched intact spinal cord showed similar CS-56 immunoreactivity (data not shown), suggesting that CSPGs in transplants are associated with developmental processes, but not induced by spinal injury. Strong expression of CSPGs (as detected by CS-56 immunostaining) was also observed inside the grafted fetal brain tissues either in neonatal or adult host (Petit et al., 2002). These findings are not consistent with the previous study that examined expression of CSPG immunoreactivity following contusive SCI and embryonic spinal cord transplantation with a different marker (Lemons et al., 1999). Using an antibody that can specifically detect chondroitin- 6 sulfate GAGs after digestion with chondroitinase ABC (tissue sections were pretreated with the enzyme in their study), they reported that transplanted embryonic spinal cord tissue contained a barely detectable amount of CSPGs at the time of transplantation and 4 days after, whereas spinal injury led to an accumulation of a high amount of CSPGs in the host cord. It is possible that CSPGs in the embryonic CNS tissue may have a different composition of chondroitin sulfate GAGs (CSGAGs) compared to injury-induced CSPGs in the host. For example, CSPGs detected in large quantity (as CS-56 immunoreactivity) inside transplants of the current study may be composed of CS-GAG species other than condroitin- 6 sulfate, whereas CSPGs in the injured host cord may contain the chondroitin- 6 sulfate. Recent studies indicated that CS-GAGs are differentially upregulated after injury, and different CS-GAGs have different potency in inhibitory activity on axonal growth (Gilbert et al., 2005; Properzi et al., 2005). The robust axonal growth into CS-56-immunoreactive transplants in neonatal hosts reported from our previous studies (Bregman, 1987b; Diener and Bregman, 1998a) and others (Petit et al., 2002) implies that the species of CS-GAGs inside transplants may not be associated with the potent inhibitory activity on axonal regeneration.

Immunostaining with a 2B6 monoclonal antibody following C'ase treatment revealed a sharp immunoreactivity along the border between host spinal cord tissue and transplanted embryonic spinal cord, indicating a deposition of a high amount of CSPGs at the exact hosttransplant interface. This phenomenon may be related to an increase in astroglial reactions (Fig. 2E) and a more profuse infiltration of inflammatory cells (Fig. 1E) along the interface. Direct apposition between mature host and immature transplanted cells may elicit astroglial and/or inflammatory reactions, leading to a deposition of a locally large amount of CSPGs at the interface. For example, in vitro interaction between astrocytes and Schwann cells resulted in increased CSPGs expression (Lakatos et al., 2000). It is likely, therefore, that the local deposition of CSPGs at the interface may occur in various transplantation strategies that inevitably involve interactions between different cell types. The above finding in the current study suggests that in addition to CSPGs-containing host spinal cord surrounding transplant, growing axons may be further challenged by the inhibitory interface, making it harder to grow into or out of transplant.

Behavioral analysis indicated that C'ase in combination with transplantation improved fine control of distal forelimb and skilled motor function such as sticker removal 
and grid walk. Fine motor control and skilled motor behaviors may be more dependent on supraspinal influence than rhythmic alternating movement such as open field locomotion triggered by the local pattern generators (Whishaw et al., 1993; Diener and Bregman, 1998b; Schucht et al., 2002). Transplantation of embryonic spinal cord tissue after neonatal SCI enhances recovery of skilled locomotion and forelimb target-reaching (Kunkel-Bagden and Bregman, 1990; Diener and Bregman, 1998b), probably by reestablishing disrupted supraspinal inputs to the spinal circuitry (Diener and Bregman, 1998a). Transplantation into adults, however, was not sufficient for substantial recovery of skilled forelimb reaching without exogenous neurotrophic support (Bregman et al., 2002). Our finding that C'ase injection following transplantation improved skilled motor behaviors in adults suggests that degradation of CSPGs may enable embryonic transplants in the adult host to reestablish disrupted supraspinal connections to the spinal motor network.

In the presence of embryonic transplants, we showed that degradation of CSPGs facilitated serotonergic innervation to the cervical motor region caudal to the injury, providing direct evidence to the above suggestion. C'ase treatment may enable the transplant to bridge the growth of regenerating raphespinal axons to the caudal cord. It is still possible that uninjured serotonergic axons descending on the contralateral side may increase the extent of bilateral projections (Bregman, 1987a). Since C'ase alone failed to significantly increase the serotonergic innervation to the right caudal cord, contribution of the bilateral projections from the contralateral side may be insignificant. Alternatively, sprouting of bilateral projections may be promoted synergistically by C'ase and trophic supports provided by transplant. Another potential mechanism of regaining supraspinal control would be a "relay" of supraspinal input by transplanted neurons to the caudal spinal cord (Bregman et al., 1993). Transplanted neurons develop mature synaptic structures and establish extensive axonal connections with the host tissue (Reier et al., 1986). Although the functional significance of this connectivity has not been determined, an increase in the number of outgoing axons from transplanted neurons, together with an increase in axonal growth into transplant, implies that synaptic communication is facilitated between supraspinal motor centers and local spinal circuitry through the increased anatomical connections. It is possible that the formation of new intraspinal connections through transplant, being promoted by degradation of CSPGs, contributes to the improved functional recovery (Bareyre et al., 2004).

An interesting observation was that C'ase alone in animals without transplants failed to improve functional recovery, contrary to the previous studies that demonstrated significant functional improvement. (Bradbury et al., 2002; Yick et al., 2004; Caggiano et al., 2005). The most plausible explanation would be that an overhemisection injury that removes more than two-thirds of the transverse area of the spinal cord does not spare enough tissue for the regenerating axons to grow through, when compared to the lesions limited to the dorsal columns (Bradbury et al., 2002) or hemicord (Yick et al., 2004). In addition, our lesion paradigm results in huge cavitation at the injury site, which is not expected in a crush lesion (Bradbury et al., 2002) or forceps compression injury (Caggiano et al., 2005). A fluid-filled cyst frequently forms at the injury site in human SCI (Bunge et al., 1997; Tuszynski et al., 1999), emphasizing the importance of the transplantation strategy.

In contrast to the raphespinal axons, we could not find any significant effect of C'ase treatment on the corticospinal axons. Neuronal populations differ in their sensitivity to inhibitory properties of CSPGs (Snow and Letourneau, 1992). For example, raphespinal and corticospinal axons showed a different capacity for regeneration into the CSPGs-rich connective tissue matrix (Inman and Steward, 2003). Since activated macrophages from a disrupted blood-brain barrier presumably initiate CSPGs upregulation (Fitch and Silver, 1997; Fitch et al., 1999), increased CSPGs may be concentrated around the blood vessels. Since serotonergic fibers innervate CNS blood vessels (Reinhard et al., 1979; Chang et al., 1989), removal of inhibitory properties of excess CSPGs might be particularly effective for the raphespinal axons growing along the blood vessels.

Our data suggest that axonal growth of transplanted neurons is also affected by inhibitory properties of CSPGs. Embryonic neurons established more frequent and longer connections with host tissue when transplanted into neonates (Reier et al., 1986; Diener and Bregman, 1998a). Since the intrinsic growth capability of transplanted neurons should be the same regardless of host age, differences in axonal growth could be attributed to the differences in host environment. Unlike the myelin-associated glycoprotein, which promotes axonal growth in embryonic neurons and inhibits in adult neurons (Mukhopadhyay et al., 1994; Cai et al., 2001), CSPGs are inhibitory to both embryonic and adult axons (Snow et al., 1990; Silver and Miller, 2004). Thus, CSPGs deposited around the transplant may be inhibitory to the axonal growth of both host and immature transplanted neurons. Recent studies showed that axonal growth of transplanted neurons is vulnerable to the host environment. Ablation of intermediate filaments in reactive astrocytes increased neurite extension of transplanted retinal cells (Kinouchi et al., 2003). Axonal growth of transplanted embryonic stem cell-derived motor neurons was affected by myelin inhibition (Harper et al., 2004). Modulation of the host environment such as degradation of CSPGs may improve connectivity of transplanted neurons with host targets.

In summary, this study suggests that CSPGs deposited around the transplant in adults inhibit axonal interaction between the host and transplant, and limit the extent of functional recovery following transplantation after SCI. We propose that the combinatorial strategy to degrade CSPGs following transplantation after SCI can increase the extent of transplant-mediated axonal remodeling and functional recovery. This strategy would be particularly beneficial to neural transplantation where removal of the inhibitory properties of CSPGs promotes axonal remodeling of both host and transplanted neurons.

\section{ACKNOWLEDGMENT}

We thank Drs. Thomas Finn and Linda MacArthur for thoughtful comments on the article.

\section{LITERATURE CITED}

Asher RA, Morgenstern DA, Fidler PS, Adcock KH, Oohira A, Braistead JE, Levine JM, Margolis RU, Rogers JH, Fawcett JW. 2000. Neurocan 
is upregulated in injured brain and in cytokine-treated astrocytes. J Neurosci 20:2427-2438.

Asher RA, Morgenstern DA, Moon LD, Fawcett JW. 2001. Chondroitin sulphate proteoglycans: inhibitory components of the glial scar. Prog Brain Res 132:611-619.

Bareyre FM, Kerschensteiner M, Raineteau O, Mettenleiter TC, Weinmann O, Schwab ME. 2004. The injured spinal cord spontaneously forms a new intraspinal circuit in adult rats. Nat Neurosci 7:269-277.

Barrett CP, Donati EJ, Guth L. 1984. Differences between adult and neonatal rats in their astroglial response to spinal injury. Exp Neurol 84:374-385.

Bradbury EJ, Moon LD, Popat RJ, King VR, Bennett GS, Patel PN, Fawcett JW, McMahon SB. 2002. Chondroitinase ABC promotes functional recovery after spinal cord injury. Nature 416:636-640.

Bregman BS. 1987a. Development of serotonin immunoreactivity in the rat spinal cord and its plasticity after neonatal spinal cord lesions. Brain Res 431:245-263.

Bregman BS. 1987b. Spinal cord transplants permit the growth of serotonergic axons across the site of neonatal spinal cord transection. Brain Res 431:265-279.

Bregman BS. 1998. Regeneration in the spinal cord. Curr Opin Neurobiol 8:800-807.

Bregman BS, McAtee M. 1993. Embryonic CNS tissue transplantation for studies of development and regeneration. Neuroprotocols 3:17-27.

Bregman BS, Kunkel-Bagden E, McAtee M, O’Neill A. 1989. Extension of the critical period for developmental plasticity of the corticospinal pathway. J Comp Neurol 282:355-370.

Bregman BS, Kunkel-Bagden E, Reier PJ, Dai HN, McAtee M, Gao D. 1993. Recovery of function after spinal cord injury: mechanisms underlying transplant-mediated recovery of function differ after spinal cord injury in newborn and adult rats. Exp Neurol 123:3-16.

Bregman BS, McAtee M, Dai HN, Kuhn PL. 1997. Neurotrophic factors increase axonal growth after spinal cord injury and transplantation in the adult rat. Exp Neurol 148:475-494.

Bregman BS, Coumans JV, Dai HN, Kuhn PL, Lynskey J, McAtee M, Sandhu F. 2002. Transplants and neurotrophic factors increase regeneration and recovery of function after spinal cord injury. Prog Brain Res 137:257-273.

Bunge MB. 2001. Bridging areas of injury in the spinal cord. Neuroscientist 7:325-339.

Bunge RP, Puckett WR, Hiester ED. 1997. Observations on the pathology of several types of human spinal cord injury, with emphasis on the astrocyte response to penetrating injuries. Adv Neurol 72:305-315.

Caggiano AO, Zimber MP, Ganguly A, Blight AR, Gruskin EA. 2005 Chondroitinase $\mathrm{ABCI}$ improves locomotion and bladder function following contusion injury of the rat spinal cord. J Neurotrauma 22:226-239.

Cai D, Qiu J, Cao Z, McAtee M, Bregman BS, Filbin MT. 2001. Neuronal cyclic AMP controls the developmental loss in ability of axons to regenerate. J Neurosci 21:4731-4739.

Camand E, Morel MP, Faissner A, Sotelo C, Dusart I. 2004. Long-term changes in the molecular composition of the glial scar and progressive increase of serotoninergic fibre sprouting after hemisection of the mouse spinal cord. Eur J Neurosci 20:1161-1176.

Chang JY, Ekblad E, Kannisto P, Owman C. 1989. Serotonin uptake into cerebrovascular nerve fibers of rat, visualization by immunohistochemistry, disappearance following sympathectomy, and release during electrical stimulation. Brain Res 492:79-88.

Chau CH, Shum DK, Li H, Pei J, Lui YY, Wirthlin L, Chan YS, Xu XM. 2004. Chondroitinase ABC enhances axonal regrowth through Schwann cell-seeded guidance channels after spinal cord injury. FASEB J 18:194-196.

Cheng H, Cao Y, Olson L. 1996. Spinal cord repair in adult paraplegic rats: partial restoration of hind limb function. Science 273:510-513.

Couchman JR, Caterson B, Christner JE, Baker JR. 1984. Mapping by monoclonal antibody detection of glycosaminoglycans in connective tissues. Nature 307:650-652.

Coumans JV, Lin TT, Dai HN, MacArthur L, McAtee M, Nash C, Bregman BS. 2001. Axonal regeneration and functional recovery after complete spinal cord transection in rats by delayed treatment with transplants and neurotrophins. J Neurosci 21:9334-9344.

Davies SJ, Field PM, Raisman G. 1996. Regeneration of cut adult axons fails even in the presence of continuous aligned glial pathways. Exp Neurol 142:203-216.

Davies SJ, Goucher DR, Doller C, Silver J. 1999. Robust regeneration of adult sensory axons in degenerating white matter of the adult rat spinal cord. J Neurosci 19:5810-5822.

Diener PS, Bregman BS. 1998a. Fetal spinal cord transplants support growth of supraspinal and segmental projections after cervical spinal cord hemisection in the neonatal rat. J Neurosci 18:779-793.

Diener PS, Bregman BS. 1998b. Fetal spinal cord transplants support the development of target reaching and coordinated postural adjustments after neonatal cervical spinal cord injury. J Neurosci 18:763-778.

Dou CL, Levine JM. 1994. Inhibition of neurite growth by the NG2 chondroitin sulfate proteoglycan. J Neurosci 14:7616-7628.

Fawcett JW, Asher RA. 1999. The glial scar and central nervous system repair. Brain Res Bull 49:377-391.

Firkins SS, Bates CA, Stelzner DJ. 1993. Corticospinal tract plasticity and astroglial reactivity after cervical spinal injury in the postnatal rat. Exp Neurol 120:1-15

Fitch MT, Silver J. 1997. Activated macrophages and the blood-brain barrier: inflammation after CNS injury leads to increases in putative inhibitory molecules. Exp Neurol 148:587-603.

Fitch MT, Doller C, Combs CK, Landreth GE, Silver J. 1999. Cellular and molecular mechanisms of glial scarring and progressive cavitation: in vivo and in vitro analysis of inflammation-induced secondary injury after CNS trauma. J Neurosci 19:8182-8198.

Fouad K, Schnell L, Bunge MB, Schwab ME, Liebscher T, Pearse DD 2005. Combining Schwann cell bridges and olfactory-ensheathing glia grafts with chondroitinase promotes locomotor recovery after complete transection of the spinal cord. J Neurosci 25:1169-1178.

Gilbert RJ, McKeon RJ, Darr A, Calabro A, Hascall VC, Bellamkonda RV. 2005. CS-4,6 is differentially upregulated in glial scar and is a potent inhibitor of neurite extension. Mol Cell Neurosci 29:545-558.

Horner PJ, Gage FH. 2000. Regenerating the damaged central nervous system. Nature 407:963-970.

Houweling DA, Bar PR, Gispen WH, Joosten EA. 1998. Spinal cord injury: bridging the lesion and the role of neurotrophic factors in repair. Prog Brain Res 117:455-471.

Hsu JY, Stein SA, Xu XM. 2005. Temporal and spatial distribution of growth-associated molecules and astroglial cells in the rat corticospinal tract during development. J Neurosci Res 80:330-340.

Inman DM, Steward O. 2003. Ascending sensory, but not other long-tract axons, regenerate into the connective tissue matrix that forms at the site of a spinal cord injury in mice. J Comp Neurol 462:431-449.

Jakeman LB, Reier PJ. 1991. Axonal projections between fetal spinal cord transplants and the adult rat spinal cord: a neuroanatomical tracing study of local interactions. J Comp Neurol 307:311-334.

Jones LL, Yamaguchi Y, Stallcup WB, Tuszynski MH. 2002. NG2 is a major chondroitin sulfate proteoglycan produced after spinal cord injury and is expressed by macrophages and oligodendrocyte progenitors. J Neurosci 22:2792-2803.

Jones LL, Margolis RU, Tuszynski MH. 2003a. The chondroitin sulfate proteoglycans neurocan, brevican, phosphacan, and versican are differentially regulated following spinal cord injury. Exp Neurol 182:399411.

Jones LL, Sajed D, Tuszynski MH. 2003b. Axonal regeneration through regions of chondroitin sulfate proteoglycan deposition after spinal cord injury: a balance of permissiveness and inhibition. J Neurosci 23:92769288.

Kato M. 1992. Walking of cats on a grid: performance of locomotor task in spinal intact and hemisected cats. Neurosci Lett 145:129-132.

Keyvan-Fouladi N, Raisman G, Li Y. 2003. Functional repair of the corticospinal tract by delayed transplantation of olfactory ensheathing cells in adult rats. J Neurosci 23:9428-9434.

Kinouchi R, Takeda M, Yang L, Wilhelmsson U, Lundkvist A, Pekny M, Chen DF. 2003. Robust neural integration from retinal transplants in mice deficient in GFAP and vimentin. Nat Neurosci 6:863-868.

Kunkel-Bagden E, Bregman BS. 1990. Spinal cord transplants enhance the recovery of locomotor function after spinal cord injury at birth. Exp Brain Res 81:25-34.

Kunkel-Bagden E, Dai HN, Bregman BS. 1993. Methods to assess the development and recovery of locomotor function after spinal cord injury in rats. Exp Neurol 119:153-164.

Lakatos A, Franklin RJ, Barnett SC. 2000. Olfactory ensheathing cells and Schwann cells differ in their in vitro interactions with astrocytes. Glia $32: 214-225$

Lakatos A, Barnett SC, Franklin RJ. 2003. Olfactory ensheathing cells induce less host astrocyte response and chondroitin sulphate proteo- 
glycan expression than Schwann cells following transplantation into adult CNS white matter. Exp Neurol 184:237-246.

Lemons ML, Howland DR, Anderson DK. 1999. Chondroitin sulfate proteoglycan immunoreactivity increases following spinal cord injury and transplantation. Exp Neurol 160:51-65.

Liu Y, Kim D, Himes BT, Chow SY, Schallert T, Murray M, Tessler A, Fischer I. 1999. Transplants of fibroblasts genetically modified to express BDNF promote regeneration of adult rat rubrospinal axons and recovery of forelimb function. J Neurosci 19:4370-4387.

McDonald JW, Liu XZ, Qu Y, Liu S, Mickey SK, Turetsky D, Gottlieb DI, Choi DW. 1999. Transplanted embryonic stem cells survive, differentiate and promote recovery in injured rat spinal cord. Nat Med 5:1410 1412.

McKeon RJ, Schreiber RC, Rudge JS, Silver J. 1991. Reduction of neurite outgrowth in a model of glial scarring following CNS injury is correlated with the expression of inhibitory molecules on reactive astrocytes. J Neurosci 11:3398-3411.

Miya D, Giszter S, Mori F, Adipudi V, Tessler A, Murray M. 1997. Fetal transplants alter the development of function after spinal cord transection in newborn rats. J Neurosci 17:4856-4872.

Moon LD, Asher RA, Rhodes KE, Fawcett JW. 2001. Regeneration of CNS axons back to their target following treatment of adult rat brain with chondroitinase ABC. Nat Neurosci 4:465-466.

Nakamura M, Houghtling RA, MacArthur L, Bayer BM, Bregman BS 2003. Differences in cytokine gene expression profile between acute and secondary injury in adult rat spinal cord. Exp Neurol 184:313-325.

Nikulina E, Tidwell JL, Dai HN, Bregman BS, Filbin MT. 2004. The phosphodiesterase inhibitor rolipram delivered after a spinal cord lesion promotes axonal regeneration and functional recovery. Proc Natl Acad Sci U S A 101:8786-8790.

Petit A, Quenneville N, Vallee A, Pierret P, Doucet G. 2002. Differences in host serotonin innervation of intrastriatal grafts are not determined by a glial scar or chondroitin sulfate proteoglycans. Exp Neurol 177:61-74.

Plant GW, Bates ML, Bunge MB. 2001. Inhibitory proteoglycan immunoreactivity is higher at the caudal than the rostral Schwann cell grafttransected spinal cord interface. Mol Cell Neurosci 17:471-487.

Properzi F, Carulli D, Asher RA, Muir E, Camargo LM, van Kuppevelt TH, ten Dam GB, Furukawa Y, Mikami T, Sugahara K, Toida T, Geller HM, Fawcett JW. 2005. Chondroitin 6-sulphate synthesis is up-regulated in injured CNS, induced by injury-related cytokines and enhanced in axon-growth inhibitory glia. Eur J Neurosci 21:378-390.

Raisman G. 2001. Olfactory ensheathing cells — another miracle cure for spinal cord injury? Nat Rev Neurosci 2:369-375.

Ramon-Cueto A, Cordero MI, Santos-Benito FF, Avila J. 2000. Functional recovery of paraplegic rats and motor axon regeneration in their spinal cords by olfactory ensheathing glia. Neuron 25:425-435.

Reier PJ, Bregman BS, Wujek JR. 1986. Intraspinal transplantation of embryonic spinal cord tissue in neonatal and adult rats. J Comp Neurol 247:275-296.

Reinhard JF Jr, Liebmann JE, Schlosberg AJ, Moskowitz MA. 1979. Serotonin neurons project to small blood vessels in the brain. Science 206:85-87.

Schrimsher GW, Reier PJ. 1993. Forelimb motor performance following dorsal column, dorsolateral funiculi, or ventrolateral funiculi lesions of the cervical spinal cord in the rat. Exp Neurol 120:264-276.

Schucht P, Raineteau O, Schwab ME, Fouad K. 2002. Anatomical correlates of locomotor recovery following dorsal and ventral lesions of the rat spinal cord. Exp Neurol 176:143-153.

Schwab ME. 2002. Repairing the injured spinal cord. Science 295:10291031.

Snow DM, Letourneau PC. 1992. Neurite outgrowth on a step gradient of chondroitin sulfate proteoglycan (CS-PG). J Neurobiol 23:322-336.

Steinmetz MP, Horn KP, Tom VJ, Miller JH, Busch SA, Nair D, Silver DJ, Silver J. 2005. Chronic enhancement of the intrinsic growth capacity of sensory neurons combined with the degradation of inhibitory proteoglycans allows functional regeneration of sensory axons through the dorsal root entry zone in the mammalian spinal cord. J Neurosci 25:8066-8076

Tang X, Davies JE, Davies S.J. 2003. Changes in distribution, cell associations, and protein expression levels of NG2, neurocan, phosphacan, brevican, versican V2, and tenascin-C during acute to chronic maturation of spinal cord scar tissue. J Neurosci Res 71:427-444.

Tuszynski MH, Gabriel K, Gerhardt K, Szollar S. 1999. Human spinal cord retains substantial structural mass in chronic stages after injury. J Neurotrauma 16:523-531.

Ughrin YM, Chen ZJ, Levine JM. 2003. Multiple regions of the NG2 proteoglycan inhibit neurite growth and induce growth cone collapse. J Neurosci 23:175-186.

Whishaw IQ, Pellis SM, Gorny B, Kolb B, Tetzlaff W. 1993. Proximal and distal impairments in rat forelimb use in reaching follow unilateral pyramidal tract lesions. Behav Brain Res 56:59-76.

Xu XM, Chen A, Guenard V, Kleitman N, Bunge MB. 1997. Bridging Schwann cell transplants promote axonal regeneration from both the rostral and caudal stumps of transected adult rat spinal cord. J Neurocytol 26:1-16.

Yick LW, So KF, Cheung PT, Wu WT. 2004. Lithium chloride reinforces the regeneration-promoting effect of chondroitinase $\mathrm{ABC}$ on rubrospinal neurons after spinal cord injury. J Neurotrauma 21:932-943. 